\title{
Formation of a galaxy with a central black hole in the Lemaître-Tolman model
}

\author{
Andrzej Krasiński \\ N. Copernicus Astronomical Center, \\ Polish Academy of Sciences, \\ Bartycka 18, 00716 Warszawa, Poland* \\ Charles Hellaby \\ Department of Mathematics and Applied Mathematics, \\ University of Cape Town, Rondebosch 7701, South Africd
}

(Dated: 25/9/2003)

\begin{abstract}
We construct two models of the formation a galaxy with a central black hole, starting from a small initial fluctuation at recombination. This is an application of previously developed methods to find a Lemaître-Tolman model that evolves from a given initial density or velocity profile to a given final density profile. We show that the black hole itself could be either a collapsed object, or a non-vacuum generalisation of a full Schwarzschild-Kruskal-Szekeres wormhole. Particular attention is paid to the black hole's apparent and event horizons.

PACS numbers: 98.80.-k, 98.62.Ai, 98.62.Js

Keywords: Cosmology, Formation of galaxies, Black holes
\end{abstract}

\section{AIM AND MOTIVATION}

It has become generally accepted that most large galaxies contain central black holes (e.g. [1]-[19]). This is due to mounting evidence for very high luminosities within very small radii at the centre of our galaxy and many others, as well as high orbital velocities of stars very close to the centre, and is bolstered by observations of radio and optical jets, as well as Seyfert galaxies and quasars at large redshifts. Since the 'very small' radii accessible by current observations are still well outside the horizons of the putative black holes, their exact nature, and even their existence, is open to debate. However, since the mean density inside the horizon of a black hole is $\rho_{s}=3 c^{6} / 32 \pi G^{3} M_{s}^{2}=1.845 \times 10^{17} /\left(M_{s} / M_{\odot}\right)^{2} \mathrm{~g} / \mathrm{cm}^{3}$, it is difficult to see how black hole collapse can be avoided above $10^{7} M_{\odot}$.

The Lemaître-Tolman (L-T) model describes the behaviour of a spherically symmetric dust distribution and has been a very fruitful source of models of inhomogenous cosmology, smaller scale structure formation, and even black holes and naked singularities.

In paper I [20], we considered the problem of finding a spherically symmetric model that evolved from a given initial density profile to a given final density profile. We showed that this can always be done with an L-T model, and we developed an alogorithm to find the arbitrary functions of such an L-T model from the given profiles.

\footnotetext{
*Electronic address: akr@camk.edu.pl

${ }^{\dagger}$ Electronic address: cwh@maths.uct.ac.za
}

A numerical example produced an Abell cluster from a density fluctuation at recombination.

In paper II 21], we generalised to finding L-T models that evolve from a given velocity profile to a given density profile, the converse, and also between two velocity profiles. Several numerical examples, including the evolution of a void, demostrated the usefulness of the method.

We now utilise these methods to consider the formation of a galaxy with a central black hole, a task for which the L-T model is particularly well suited. Although spiral galaxies are not exactly spherically symmetric, both the core and the halo - together containing more mass than the disk - are quite close to it, so the L-T model is not a bad first approximation.

The present state of the galaxy is defined by a mass distribution that consists of two parts:

1. The part outside the apparent horizon at $t_{2}-$ for which we use an approximation to the observationally determined density profile of the M87 galaxy. This part extends inward to a sphere of mass $M_{B H}$, where $M_{B H}$ is the observationally determined mass of the black hole in the M87 galaxy.

2. The part inside the apparent horizon at $t_{2}$. Since, for fundamental reasons, no observational data at all exist for this region apart from the value of $M_{B H}$, we were free to choose any geometry. We chose two examples:

2a. A simple subcase of the $\mathrm{L}-\mathrm{T}$ model, discussed in sec. III F as an illustrative example of properties of horizons. In this model, the black hole does not exist initially and is formed in the course of evolution.

2b. A pre-existing wormhole, also chosen arbitrarily for simplicity of the calculations.

The boundary between the "inside" and "outside" at 
times other than $t_{2}$ goes along a comoving mass shell, so that at $t<t_{2}$ the apparent horizon resides in the inside part.

For the initial state, at $t_{1}=$ (the last scattering of the cosmic microwave background (CMB) radiation), no usable observational data are available, either, but hopefully only temporarily. The expected angular size on the CMB sky of a perturbation that will develop into a single galaxy $\left(0.004^{\circ}\right)$ is much smaller than the current best resolution $\left(0.2^{\circ}\right)$. Therefore we tried an exactly homogeneous initial density and a homogeneous initial velocity. The former turned out to lead to an unacceptable configuration at $t_{2}$ : a collapsing hyperbolic model with no Big Bang in the past. Consequently, we settled on the homogeneous initial velocity, which then implied the amplitude below $10^{-5}$ for the initial density perturbation.

These two states, at $t_{1}$ and $t_{2}$, uniquely define the $\mathrm{L}^{-}$ $\mathrm{T}$ model that evolves between them, as shown in Paper II. The 3-d surface graphs of density as a function of mass and time show that the evolution proceeds without shell crossings, and so the model is acceptable, at least qualitatively.

\section{BASIC PROPERTIES OF THE LEMAÎTRE-TOLMAN MODEL}

The Lemaître-Tolman (L-T) model 22, 23] is a spherically symmetric nonstatic solution of the Einstein equations with a dust source. See 24] for an extensive list of properties and other work on this model. Its metric is:

$$
\mathrm{d} s^{2}=\mathrm{d} t^{2}-\frac{\left(R^{\prime}\right)^{2}}{1+2 E(r)} \mathrm{d} r^{2}-R^{2}(t, r)\left(\mathrm{d} \vartheta^{2}+\sin ^{2} \vartheta \mathrm{d} \varphi^{2}\right),
$$

where $E(r)$ is an arbitrary function of integration, $R^{\prime}=$ $\partial R / \partial r$, and $R(t, r)$ obeys

$$
\dot{R}^{2}=2 E+\frac{2 M}{R}+\frac{\Lambda}{3} R^{2},
$$

where $\dot{R}=\partial R / \partial t$ and $\Lambda$ is the cosmological constant. Eq. (2.2) is a first integral of the Einstein equations, and $M=M(r)$ is another arbitrary function of integration. The mass-density is:

$$
\kappa \rho=\frac{2 M^{\prime}}{R^{2} R^{\prime}}, \quad \text { where } \kappa=\frac{8 \pi G}{c^{4}} .
$$

In the following, we will assume $\Lambda=0$. Then eq. (2.2) can be solved explicitly, and the solutions are: when $E<$
0 (elliptic evolution):

$$
\begin{gathered}
R(t, r)=\frac{M}{(-2 E)}(1-\cos \eta), \\
\eta-\sin \eta=\frac{(-2 E)^{3 / 2}}{M}\left(t-t_{B}(r)\right),
\end{gathered}
$$

where $\eta$ is a parameter; when $E=0$ (parabolic evolution):

$$
R(t, r)=\left[\frac{9}{2} M\left(t-t_{B}(r)\right)^{2}\right]^{1 / 3},
$$

and when $E>0$ (hyperbolic evolution):

$$
\begin{gathered}
R(t, r)=\frac{M}{2 E}(\cosh \eta-1), \\
\sinh \eta-\eta=\frac{(2 E)^{3 / 2}}{M}\left(t-t_{B}(r)\right),
\end{gathered}
$$

where $t_{B}(r)$ is one more arbitrary integration function (the bang time). Note that all the formulae given so far are covariant under arbitrary coordinate transformations $\tilde{r}=g(r)$, and so $r$ can be chosen at will. This means one of the three functions $E(r), M(r)$ and $t_{B}(r)$ can be fixed at our convenience by the appropriate choice of $g$. We can define a scale radius and a scale time for each worldline with

$$
\begin{aligned}
& P(r)=\frac{2 M}{|2 E|} \\
& T(r)=\frac{2 \pi M}{|2 E|^{3 / 2}}
\end{aligned}
$$

and it is evident from (2.4) that, for the elliptic case, these are the maximum $R$ and the lifetime for each $r$ value. The crunch time is then

$$
t_{C}(r)=t_{B}(r)+T(r) .
$$

Writing eq. 2.4b) at $\eta=2 \pi$, where $t=t_{C}$, i.e. at the Big Crunch, and then dividing the two equations we obtain

$$
\eta-\sin \eta=2 \pi\left(t-t_{B}\right) /\left(t_{C}-t_{B}\right)
$$

so larger $\eta$ means only that the dust particle has completed a larger fraction of its lifetime between the Bang and the Crunch.

The parametric solutions (2.4) and (2.6) can also be written 


$$
\begin{array}{ll}
t=t_{B}+\frac{M}{(-2 E)^{3 / 2}}\left\{\arccos \left(1+\frac{2 E R}{M}\right)-2 \sqrt{\frac{-E R}{M}\left(1+\frac{E R}{M}\right)}\right\}, \quad 0 \leq \eta \leq \pi, \\
t=t_{B}+\frac{M}{(-2 E)^{3 / 2}}\left\{\pi+\arccos \left(-1-\frac{2 E R}{M}\right)+2 \sqrt{\frac{-E R}{M}\left(1+\frac{E R}{M}\right)}\right\}, \quad \pi \leq \eta \leq 2 \pi,
\end{array}
$$

for the expanding and collapsing elliptic cases, and

$$
\begin{array}{r}
t=t_{B}+\frac{M}{(2 E)^{3 / 2}}\left\{\sqrt{\frac{2 E R}{M}\left(2+\frac{2 E R}{M}\right)}\right. \\
\left.-\operatorname{arcosh}\left(1+\frac{2 E R}{M}\right)\right\}
\end{array}
$$

for the hyperbolic case (expanding).

Apart from extended parabolic regions, there are also parabolic boundaries between elliptic and hyperbolic regions, where $E \rightarrow 0$, but $E^{\prime} \neq 0$. The limiting forms of equations (2.4) and (2.6) are found by requiring well behaved time evolution and setting

$$
\eta=\tilde{\eta} \sqrt{E}
$$

so that $\tilde{\eta}$ is finite if $\left(t-t_{B}\right)$ is.

The Friedmann models are contained in the L-T class as the limit:

$$
t_{B}=\text { const }, \quad|E|^{3 / 2} / M=\text { const },
$$

and one of the standard radial coordinates for the Friedmann model results if the coordinates in (2.4) - 2.6) are additionally chosen so that:

$$
M=M_{0} r^{3} \quad \rightarrow \quad E=E_{0} r^{2}
$$

with $M_{0}$ and $E_{0}$ being constants.

In constructing our galaxy model, it will be convenient to use $M(r)$ as the radial coordinate (i.e. $\tilde{r}=M(r)$ ) - because in most sections we shall not need to pass through any "necks" or "bellies". Thus, $M(r)$ will be a strictly growing function in the whole region under consideration. In some of the sections we shall consider a black hole with a "neck" or "wormhole", but even there, because of spherical symmetry, we will consider only one side of the wormhole, where $M(r)$ is also increasing.

Then with $R=R(t, M)$ :

$$
\kappa \rho=\frac{2}{R^{2} \frac{\partial R}{\partial M}} \equiv \frac{6}{\frac{\partial\left(R^{3}\right)}{\partial M}} .
$$

In the present paper we will apply the L-T model to a problem related to that considered in papers I \& II:
Connecting, by an L-T evolution, an initial state of the Universe, defined by a mass-density or velocity distribution, to a final state defined by a density distribution that contains a black hole.

\section{A. Origin conditions}

An origin, or centre of spherical symmetry, occurs at $r=r_{c}$ if $R\left(t, r_{c}\right)=0$ for all $t$. The conditions for a regular centre have been derived in 25] from the requirements that, away from the bang and crunch, and in the limit $r \rightarrow r_{c}$ :

- $\eta$ in (2.4) and (2.6) must be finite if $\left(t-t_{B}\right)$ is finite,

- the density (2.3) and the Kretschmann scalar are non-divergent, and the density is not zero,

- on a constant time slice $d \rho / d R=0$.

In the equations below, the symbol $O_{d}(M)$ will denote a function that has the property $\lim _{M \rightarrow 0}\left(O_{d}(M) / M^{d}\right)=$ 0 . The resulting conditions for the neighbourhood of $r_{c}$ are

$$
\begin{aligned}
& R=\beta(t) M^{1 / 3}+O_{1 / 3}(M) \quad \text { along constant } t \\
& E=\gamma M^{2 / 3}+O_{2 / 3}(M), \\
& t_{B}=\tau O_{c}(M), \quad c>1 / 3 \\
& \kappa \rho=6 / \beta^{3}+O_{0}(M) \\
& M\left(r_{c}\right)=0 .
\end{aligned}
$$

We also need $\tau<0$ to avoid shell crossings.

\section{B. Shell crossings, maxima and minima}

Shell crossings, where a constant $r$ shell collides with its neighbour, are loci of $R^{\prime}=0$ that are not regular maxima or minima of $R$. They create undesireable singularities where the density diverges and changes sign. The conditions on the 3 arbitrary functions that ensure none be present anywhere in an L-T model, as well as those for regular maxima and minima in spatial sections, were given in [26], and will be used below. 


\section{APPARENT AND EVENT HORIZONS IN THE L-T MODEL}

We will be modelling a galactic black hole, so it will be useful to consider its horizons. Apparent and event horizons of L-T models were studied in 27], in which L-T models that generalise the Schwarzschild-KruskalSzekeres topology to non-vacuum were demonstrated. It was shown that, when there's matter present, the light rays get even less far through the wormhole than in the vacuum case. The diversity of possible topologies was discussed. We lay out further details of the apparent horizon here.

\section{A. Definitions and basic properties}

Let us write the evolution equation (2.2) with $\Lambda=0$ as

$$
\begin{aligned}
& \dot{R}=\ell \sqrt{\frac{2 M}{R}+2 E}, \\
& \text { where }\left\{\begin{aligned}
\ell=+1 & \text { in the expanding phase, } \\
\ell=-1 & \text { in the collapsing phase. }
\end{aligned}\right.
\end{aligned}
$$

The radial light rays must be geodesics by symmetry:

$$
0=-d t^{2}+\frac{\left(R^{\prime}\right)^{2}}{1+2 E} d r^{2}
$$

and this may be written as

$$
\begin{aligned}
t_{n}^{\prime}=\left.\frac{\mathrm{d} t}{\mathrm{~d} r}\right|_{n}=\frac{j R^{\prime}}{\sqrt{1+2 E}}, \\
\text { where }\left\{\begin{array}{r}
j=+1 \\
j=-1
\end{array}\right. \text { for outgoing rays, }
\end{aligned}
$$

whose solution we write as $t=t_{n}\left(r, t_{n 0}\right)$, or often just $t_{n}(r)$ or $t_{n}$.

\section{Apparent horizons}

Along a ray we have

$$
\begin{aligned}
R_{n} & =R\left(t_{n}, r\right), \\
\left(R_{n}\right)^{\prime} & =\left.\dot{R} \frac{\mathrm{d} t}{\mathrm{~d} r}\right|_{n}+R^{\prime} \\
& =\left(\ell j \frac{\sqrt{\frac{2 M}{R}+2 E}}{\sqrt{1+2 E}}+1\right) R^{\prime} .
\end{aligned}
$$

The apparent horizon $(\mathrm{AH})$ is the hypersurface in spacetime where the rays are momentarily at constant $R$ :

$$
\begin{array}{r}
\left(R_{n}\right)^{\prime}=0 \Rightarrow \sqrt{\frac{2 M}{R}+2 E}=-\ell j \sqrt{1+2 E} \quad \Rightarrow \\
\ell j=-1, \quad \text { and } \quad R=2 M .
\end{array}
$$

There are in fact two apparent horizons:

The future $\mathrm{AH}:\left(\mathrm{AH}^{+}\right)$, where

$$
\begin{cases}j=+1 & \text { (outgoing rays) } \\ \ell=-1 & \text { (in a collapsing phase) }\end{cases}
$$

and the past $\mathrm{AH}:\left(\mathrm{AH}^{-}\right)$, where

$$
\begin{cases}j=-1 & \text { (incoming rays) } \\ \ell=+1 & \text { (in an expanding phase). }\end{cases}
$$

We find $d t / d r$ along the AH by differentiating (3.7):

$$
\dot{R} d t+R^{\prime} d r=2 M^{\prime} d r
$$

giving

$$
t_{\mathrm{AH}}^{\prime}=\left.\frac{\mathrm{d} t}{\mathrm{~d} r}\right|_{\mathrm{AH}}=\frac{2 M^{\prime}-R^{\prime}}{\dot{R}}=\frac{2 M^{\prime}-R^{\prime}}{\ell \sqrt{\frac{2 M}{R}+2 E}},
$$

and, since $R=2 M$ on the $\mathrm{AH}$,

$$
\left.\frac{\mathrm{d} t}{\mathrm{~d} r}\right|_{\mathrm{AH}}=\frac{\ell\left(2 M^{\prime}-R^{\prime}\right)}{\sqrt{1+2 E}} .
$$

In the vacuum case $\rho=0$, which implies $M^{\prime}=0$, we have $\mathrm{d} t /\left.\mathrm{d} r\right|_{\mathrm{AH}}=\mathrm{d} t /\left.\mathrm{d} r\right|_{n}$ since $\ell j=-1$. Note that $M^{\prime}=0$ could be only local, so the $\mathrm{AH}$ would only be null in that region. In the Schwarzschild metric, where $M^{\prime}=0$ everywhere, this is consistent with $R=2 M$ being the locus of the event horizons; and in this case they coincide with the apparent horizons.

Recall that in the Schwarzschild spacetime the future $\&$ past event horizons, $\mathrm{EH}^{+} \& \mathrm{EH}^{-}$, cross in the neck at the moment it is widest. (Call this event O.) This holds for L-T models too. For hyperbolic regions, with $E \geq 0$ along each dust worldline, there is either only expansion or only collapse, i.e. only one $\mathrm{AH}$ (either $\mathrm{AH}^{+}$or $\mathrm{AH}^{-}$) can occur. The AHs can thus cross only in an elliptic $E<0$ region. At the neck of a $\mathrm{L}-\mathrm{T}$ wormhole, where $2 E=-1, M$ is a minimum, and $t_{B}$ is maximum, the moment of maximum expansion is

$$
\dot{R}^{2}=0=\frac{2 M}{R}-1 \quad \rightarrow \quad R_{\max }\left(M_{\min }\right)=2 M .
$$

At all other $E$ values in an elliptic region $-1<2 E<0$, we find $\dot{R}=0 \quad \rightarrow \quad R_{\max }=2 M /(-2 E)>2 M$. Thus 
$R=2 M$ has two solutions - one in the expanding phase $\&$ one in the collapsing phase. So the $\mathrm{AH}^{+} \& \mathrm{AH}^{-}$meet at the neck maximum (event $\mathrm{O}$ ).

To establish whether $\mathrm{AH}$ is timelike, null or spacelike, we compare the slope of the $\mathrm{AH}^{+}$with the outgoing light ray (or the $\mathrm{AH}^{-}$with the incoming light ray), i.e. $\ell j=$ -1 :

$$
B=\left.\frac{\mathrm{d} t}{\mathrm{~d} r}\right|_{A H} /\left.\frac{\mathrm{d} t}{\mathrm{~d} r}\right|_{n}=-\ell j\left(1-\frac{2 M^{\prime}}{R^{\prime}}\right)=\left(1-\frac{2 M^{\prime}}{R^{\prime}}\right)
$$

but below, we will actually calculate

$$
\begin{aligned}
\bar{B}=\left(\frac{R^{\prime}}{M^{\prime}}-1\right)_{\mathrm{AH}^{ \pm}}=\left(\frac{\partial R}{\partial M}-1\right)_{\mathrm{AH}^{ \pm}} \\
=\frac{1+B}{1-B}=1-\ell \sqrt{1+2 E} \frac{\mathrm{d} t_{\mathrm{AH}}}{\mathrm{d} M}
\end{aligned}
$$

where we have used (3.11) and written $R^{\prime} / M^{\prime}=\partial R / \partial M$ and $t_{B}^{\prime} / M^{\prime}=\mathrm{d} t_{B} / \mathrm{d} M$, since $M^{\prime}>0$. Now since the conditions for no shell crossings [26] require $M^{\prime} \geq 0$ where $R^{\prime}>0$ and vice-versa, we have

$$
\begin{array}{clll}
B_{\max }=1, \quad \bar{B}=+\infty & \rightarrow \mathrm{AH}^{+} \text {outgoing null } & & \left(\text { when } M^{\prime}=0, \rho=0\right), \\
1>B>-1, \quad+\infty>\bar{B}>0 & \rightarrow \mathrm{AH}^{+} \text {spacelike } & \left(\text { for most } M^{\prime}\right), \\
B=-1, \quad \bar{B}=0 & \rightarrow \mathrm{AH}^{+} \text {incoming null } & \left(\text { for large } M^{\prime} / R^{\prime}\right), \\
-1>B>-\infty, \quad 0>\bar{B}>-1 & \rightarrow \mathrm{AH}^{+} \text {incoming timelike } & \left(\text { for very large } M^{\prime} / R^{\prime}\right),
\end{array}
$$

so an outgoing timelike $\mathrm{AH}^{+}$is not possible. This means outgoing light rays that reach the $\mathrm{AH}^{+}$always fall inside $\mathrm{AH}^{+}$, except where $M^{\prime}=0$, in which case they move along it. The possibility that $\mathrm{AH}^{ \pm}$is timelike $(\bar{B}<0)$ holds if ${ }^{1}$

$$
\ell \frac{\mathrm{d} t_{\mathrm{AH}}}{\mathrm{d} M}>\frac{1}{\sqrt{1+2 E}} .
$$

This means that the smaller $E$ is, the steeper the locus of the AH must be to make it timelike.

The argument is similar for light rays at $\mathrm{AH}^{-}$, except that 'incoming' should swopped with 'outgoing'. If ingoing light rays reach the $\mathrm{AH}^{-}$, they pass out of it or run along it.

Since this is true for every point on $\mathrm{AH}^{+} \& \mathrm{AH}^{-}$, radial light rays in dense wormholes are more trapped and go even less far than in vacuum. In particular, if ${ }^{2} \rho>0$ where $2 E=-1$, light rays starting at $\mathrm{O}$ fall inside $\mathrm{AH}^{+}$ to the future and inside $\mathrm{AH}^{-}$to the past.

${ }^{1}$ Note that eq. 3.16 can be equivalently written as follows:

$$
\ell t_{A H}^{\prime}>\frac{M^{\prime}}{\sqrt{1+2 E}}=\mathcal{M}^{\prime},
$$

where $\mathcal{M}$ is the sum of all the rest masses within the $r=$ const sphere, equal to $4 \pi \int_{0}^{r} \sqrt{-g(t, x)} \mathrm{d} x \equiv$ $4 \pi \int_{0}^{r}\left[R^{2}(t, x) R^{\prime}(t, x) / \sqrt{2 E+1}\right] \mathrm{d} x, \quad$ while $M=$ $4 \pi \int_{0}^{r} R^{2}(t, x) R^{\prime}(t, x) \mathrm{d} x$ is the active gravitational mass (see 28] for a detailed discussion).

2 Since a regular minimum or maximum $R^{\prime}=0$ requires all of $M^{\prime}$, $E^{\prime}$ and $t_{B}^{\prime}$ to be locally zero, $\rho>0 \Rightarrow M^{\prime \prime} / R^{\prime \prime}>0$.

\section{Event horizons}

The event horizon is the very last ray to reach future null infinity $\left(\mathrm{EH}^{+}\right)$, or the very first one to come in from past null infinity $\left(\mathrm{EH}^{-}\right)$. If we have vacuum $\left(M^{\prime}=0\right)$ everywhere, then light rays travel along $R=2 M$, and the EHs coincide with the AHs. If there is matter, $M^{\prime}>0$, on any worldline, then the incoming light rays emerge from $\mathrm{AH}^{-}$and outgoing light rays fall into $\mathrm{AH}^{+}$at that $r$ value, and so the EHs split off from the AHs (see 27]).

\section{B. Locating the apparent horizons in elliptic regions}

\section{1. $A H^{-}$during expansion}

We shall first consider the expansion phase of an elliptic model, where $0 \leq \eta \leq \pi$ and $E<0$, so we have $\ell=+1$ and only $\mathrm{AH}^{-}$is present. Since $R=2 M$ on an $\mathrm{AH}$, we have from (2.4a):

$$
\cos \eta_{\mathrm{AH}}=1+4 E,
$$

and thus, along a given worldline, the proper time of passing through the $\mathrm{AH}$, counted from the Bang time $t_{B}$, can be calculated from (2.11) with $R=2 M$ to be

$$
t_{\mathrm{AH}^{-}}-t_{B}=M \frac{\arccos (1+4 E)-2 \sqrt{-2 E(1+2 E)}}{(-2 E)^{3 / 2}} .
$$


The function $F=t_{\mathrm{AH}^{-}}-t_{B}$ of the argument $f=2 E$, defined in (3.18) has the following properties

$$
\begin{gathered}
F(-1)=M \pi, \quad F(0)=4 M / 3, \\
\frac{\mathrm{d} F}{\mathrm{~d} f}<0 \quad \text { for } \quad-1<f<0,
\end{gathered}
$$

i.e. it is decreasing. These properties mean that the $\mathrm{AH}$ does not touch the Big Bang anywhere except at a centre, $M=0$, even if $E=0$.

Along all worldlines with $E>-1 / 2$, even parabolic worldlines $E=0 \& M \neq 0$, the dust particles emerge from $\mathrm{AH}^{-}$a finite time after the Big Bang $(\eta=0)$ and a finite time before maximum expansion $(\eta=\pi)$. In order to find the slope of $\mathrm{AH}^{-}$, we differentiate (3.18) with respect to $M$ to obtain

$$
\begin{aligned}
& \frac{\mathrm{d} t_{\mathrm{AH}^{-}}=}{\mathrm{d} M}=\left[\frac{1}{(-2 E)^{3 / 2}}+\frac{3 M}{(-2 E)^{5 / 2}} \frac{\mathrm{d} E}{\mathrm{~d} M}\right] \arccos (1+4 E) \\
&+\frac{1}{E} \sqrt{1+2 E}-\frac{M(3+2 E)}{2 E^{2} \sqrt{1+2 E}} \frac{\mathrm{d} E}{\mathrm{~d} M}+\frac{\mathrm{d} t_{B}}{\mathrm{~d} M} .
\end{aligned}
$$

In general, this is very difficult to analyse, but for special cases it will be possible.

So although (3.17) shows wordlines with larger $E$ exit $\mathrm{AH}^{-}$at a later stage of evolution, this may not correspond to a later time $t$, or even to a longer time $\left(t-t_{B}\right)$ since the the bang. It is not at all necessary that $E$ is a monotonically decreasing function of $r$ in an elliptic region; in general it can increase and decrease again any number of times.

\section{2. $A H^{-}$near an origin}

On the $\mathrm{AH}^{-}$in the neighbourhood of a regular center, we obtain from (3.17) and (2.18b) to lowest order:

$$
1-\cos \eta_{\mathrm{AH}}=-4 \gamma M^{2 / 3}+O_{2 / 3}(M) .
$$

and consequently, $\eta_{\mathrm{AH}^{-}} \rightarrow 0$ as $r \rightarrow r_{c}$, i.e. $\mathrm{AH}^{-}$touches the Big Bang set at the center. Since $E\left(r_{c}\right)=0$ and $E<0$ in the neighbourhood of the center, it follows that $E^{\prime}\left(r_{c}\right) \leq 0$, and, via (3.17), that the dust particles with larger $r$ (smaller $E$ ) exit $\mathrm{AH}^{-}$with larger values of $\eta$.

To show the behaviour of $\mathrm{AH}^{-}$near $r_{c}$ is not unique, we take the following example for $E(M)$

$$
\begin{aligned}
E=M^{2 / 3}\left(\gamma+\gamma_{2} M^{d}\right), & \\
d & >2 / 3, \quad \gamma \neq 0, \quad \gamma_{2} \neq 0 .
\end{aligned}
$$

Putting (3.22) and (2.18c) in (3.20) we find ${ }^{3}$, neglecting powers of $M$ that are necessarily positive,

$$
\frac{\mathrm{d} t_{\mathrm{AH}^{-}}}{\mathrm{d} M} \approx c \tau M^{c-1}+\frac{4}{3}
$$

and from (3.14) with $\ell=+1$ and (3.23) we see that,

$$
\bar{B} \approx-c \tau M^{c-1}-\frac{1}{3}+\ldots
$$

The behaviour of the apparent horizon in the vicinity of the center is not unique, each of the cases listed in (3.15) can occur, depending on whether $c>1$. This nonuniqueness of behaviour is connected with the shell-focussing singularities that appear in some L-T models. Various studies [29]-37] have shown that outgoing light rays may emerge from the central point of the Big Crunch and even reach infinity. Moreover, even though, in those cases, this central point (where the Big Crunch first forms) appears to be a single point in comoving coordinates, it is in fact a finite segment of a null line in the Penrose diagram, see [29]. At the Big Bang, we have the reverse - incoming light rays may reach the central point of the Bang singularity. Any radial light ray emitted from the center of symmetry that falls into the Big Crunch must first increase its $R$ value, and then decrease, i.e. must cross the $\mathrm{AH}+$ in between. In consequence, the $\mathrm{AH}+$ cannot touch the center of $\mathrm{BC}$ earlier than the null singularity does. Similarly, the $\mathrm{AH}^{-}$cannot touch the center of $\mathrm{BB}$ later than the null singularity does.

\section{3. $A H^{-}$in the parabolic limit}

A shell of parabolic worldlines occurs at the boundary between elliptic and hyperbolic regions, where $E \rightarrow 0$, but $E^{\prime} \neq 0$ and ${ }^{4} M>0$. From (3.17), (3.18), (2.8),

${ }^{3}$ Since $M^{1 / 3}$ is a natural measure of proper radius near an origin, the slope of $\mathrm{AH}$ - is zero:

$$
\frac{\mathrm{d} t_{\mathrm{AH}-}}{\mathrm{d} M^{1 / 3}} \approx 3 c \tau M^{c-1 / 3}+4 M^{2 / 3}+\ldots \approx 0
$$

Away from the centre, the sign of $\mathrm{d} t_{\mathrm{AH}^{-}} / \mathrm{d} M^{1 / 3}$ depends on whether or not $c>1$, as $\tau<0$ for no shell crossings [26].

${ }^{4}$ Elliptic and parabolic regions are not possible for $M=0$ 
(3.20) and (3.14) with $\ell=+1$, we see that

$$
\begin{gathered}
\eta \underset{E \rightarrow 0}{\longrightarrow} 0 \\
t_{\mathrm{AH}^{-}}-t_{B} \underset{E \rightarrow 0}{\longrightarrow} \frac{4 M}{3} \\
\frac{\mathrm{d} t_{\mathrm{AH}}-}{\mathrm{d} M} \underset{E \rightarrow 0}{\underset{E \rightarrow 0}{\longrightarrow}} \frac{4}{3}-\frac{4 M}{5} \frac{\mathrm{d} E}{\mathrm{~d} M}+\frac{\mathrm{d} t_{B}}{\mathrm{~d} M} \\
\bar{B}_{\mathrm{AH}^{-}} \underset{E \rightarrow 0}{\longrightarrow}-\frac{1}{3}+\frac{4 M}{5} \frac{\mathrm{d} E}{\mathrm{~d} M}-\frac{\mathrm{d} t_{B}}{\mathrm{~d} M}
\end{gathered}
$$

so $\mathrm{AH}^{-}$never touches the bang here, despite $\eta$ being zero. The divergence of the worldline lifetime $T$ suggests that either the bang time or the crunch time recedes to infinity, as would be expected in a hyperbolic region. There is in fact a third possibility, as there is no reason why both times should not diverge. Indeed, an asymptotically flat model is achieved by letting $E \rightarrow 0$ as $r \rightarrow \infty$ [27], with both bang and crunch times diverging ${ }^{5}$. We also see the slope and the causal nature of $\mathrm{AH}^{-}$are uncertain here.

\section{4. $A H^{+}$during collapse}

This can be obtained form the above by replacing $(t-$ $\left.t_{B}\right)$ with $\left(t_{C}-t\right), \eta$ with $(2 \pi-\eta)$, flipping the signs of $\ell$ and $j$, and swopping "incoming" with "outgoing". However, keeping $t_{B}$ as our arbitrary function, we have $\pi \leq \eta \leq 2 \pi$. Eq. (3.17) still applies, but instead of (3.18) and (3.20), we now obtain

$$
\begin{aligned}
& \left(t-t_{B}\right)_{\mathrm{AH}^{+}}= \\
& M \frac{\pi+\arccos (-1-4 E)+2 \sqrt{-2 E(1+2 E)}}{(-2 E)^{3 / 2}}, \\
& \frac{\mathrm{d} t_{\mathrm{AH}}}{\mathrm{d} M}= \\
& {\left[\frac{1}{(-2 E)^{3 / 2}}+\frac{3 M}{(-2 E)^{5 / 2}} \frac{\mathrm{d} E}{\mathrm{~d} M}\right][\pi+\arccos (-1-4 E)]} \\
& -\frac{1}{E} \sqrt{1+2 E}+\frac{M(3+2 E)}{2 E^{2} \sqrt{1+2 E}} \frac{\mathrm{d} E}{\mathrm{~d} M}+\frac{\mathrm{d} t_{B}}{\mathrm{~d} M} .
\end{aligned}
$$

and (3.14) applies with $\ell=-1$. The special cases all follow in the same way. Near an origin, we again find nonunique behaviour, one of the possibilities this time being light rays escaping the crunch at $r_{c}$ before $\mathrm{AH}^{+}$ forms. At a regular extremum where $E=-1 / 2, \eta_{\mathrm{AH}^{+}} \rightarrow$ $\pi$ and $\mathrm{AH}^{+}$crosses $\mathrm{AH}^{-}$. In the parabolic limit, $\eta_{\mathrm{AH}^{+}} \rightarrow$

\footnotetext{
${ }^{5}$ Another example of this kind will be discussed in sec. IIIF
}

$$
\begin{aligned}
& 0, t_{C}- t_{\mathrm{AH}^{+}} \rightarrow 4 M / 3, \text { and } \\
& \frac{\mathrm{d} t_{\mathrm{AH}^{+}}}{\mathrm{d} M} \underset{E \rightarrow 0}{\longrightarrow}-\frac{4}{3}+\frac{4 M}{5} \frac{\mathrm{d} E}{\mathrm{~d} M}+\frac{\mathrm{d} t_{C}}{\mathrm{~d} M}, \\
& \bar{B}_{\mathrm{AH}^{+}} \underset{E \rightarrow 0}{\longrightarrow}-\frac{1}{3}+\frac{4 M}{5} \frac{\mathrm{d} E}{\mathrm{~d} M}+\frac{\mathrm{d} t_{C}}{\mathrm{~d} M},
\end{aligned}
$$

where $t_{C}$ is given by (2.9) \& (2.8).

\section{Apparent horizons in parabolic regions}

The corresponding results in an expanding $E=0$, $E^{\prime}=0$ L-T model follow from section IIIB3 (or directly from (2.5) with $R=2 M)$. In particular (3.25d) and (3.25e become

$$
\begin{aligned}
& \frac{\mathrm{d} t_{\mathrm{AH}^{-}}}{\mathrm{d} M}=\frac{4}{3}+\frac{\mathrm{d} t_{B}}{\mathrm{~d} M} \\
& \bar{B}_{\mathrm{AH}^{-}}=-\frac{1}{3}-\frac{\mathrm{d} t_{B}}{\mathrm{~d} M}
\end{aligned}
$$

Despite the no shell crossing condition $\mathrm{d} t_{B} / \mathrm{d} M<0$, $\mathrm{AH}^{-}$may still exhibit all possible behaviours of (3.15) with "outgoing" and "incoming" interchanged. In a collapsing parabolic model, time reversed results apply.

\section{Locating apparent horizons in hyperbolic regions}

Using the same methods as for expanding elliptic regions, we find the behaviour of the $\mathrm{AH}$ in expanding hyperbolic regions is qualitatively the same. There is of course only one $\mathrm{AH}$, no maximum expansion, and loci where $E=-1 / 2$ are not possible. But the results for origins and for the parabolic limit both carry over. Collapsing hyperbolic regions are essentially like collapsing elliptic regions.

\section{E. Locating the event horizon}

In general, locating the EH involves integrating (3.3), which cannot be done analytically because $R^{\prime}$ is not a simple expression. In addition, we must find the last outgoing ray to escape the crunch, or the first incoming ray to avoid the bang. For a particular model, $\mathrm{EH}^{+}$ is located numerically using a compactified coordinate representation of the spacetime in section IIIF3.

\section{F. An illustration - a simple recollapsing model}

We shall illustrate several properties of the L-T model and of its apparent horizons on a simple example. In the 
present subsection, we will use this model with unrealistic parameter values, chosen in such a way that all the figures are easily readable. Later, in sec. [V] we will use the same model for modelling a galaxy with a black hole at the center, with parameters chosen to fit observational data.

\section{Definition}

We take an $E<0$ L-T model with a regular centre, whose Big Bang function $t_{B}(M)$ is

$$
t_{B}(M)=-b M^{2}+t_{B 0},
$$

and whose Big Crunch function is

$$
t_{C}(M)=a M^{3}+T_{0}+t_{B 0},
$$

where the parameter $T_{0}$ is the lifetime of the central worldline where $M=0$. The numerical values of the parameters used in the figures will be $a=2 \cdot 10^{4}, b=200$, $t_{B 0}=5, T_{0}=0.05$. Their values were chosen so as to make the figures readable and illustrative, at this point they are unrelated to any astrophysical quantities. Since $t=t_{C}$ at $\eta=2 \pi$, we find from eq. (2.4):

$$
\begin{aligned}
E(M) & =-\frac{1}{2}\left(\frac{2 \pi M}{t_{C}-t_{B}}\right)^{2 / 3} \\
& =-\left(\frac{\pi^{2}}{2}\right)^{1 / 3} \frac{M^{2 / 3}}{\left(a M^{3}+b M^{2}+T_{0}\right)^{2 / 3}} .
\end{aligned}
$$

Note that as $M \rightarrow \infty$, we have $t_{B} \rightarrow-\infty, t_{C} \rightarrow+\infty$ and $E \rightarrow 0$. Hence, the space contains infinite mass and has infinite volume. Unlike in the Friedmann models, positive space curvature does not imply finite volume; this has been known since long ago [26, 38].

\section{Description}

The main features of this model are shown in Fig. 1 Note that the $\mathrm{AH}^{+}$first appears not at the center, but at a finite distance from the center, where the function $t_{A H^{+}}(M)$ has its minimum, and at a time $t_{h s}<t_{C}(0)$.

At all times after the crunch first forms, $t>t_{C}(0)$, the mass $M_{S}$ already swallowed up by the singularity is necessarily smaller than the mass $M_{B H}$ that disappeared into the $\mathrm{AH}^{+}$. So for an object in which the black hole already exists, the time $t=$ now must be taken after $t_{h s}$ and in Fig. 1 it is also greater than $t_{C}(0)$. The mass $M_{S}$ cannot even be estimated by astronomical methods.

The situation is similar, but reversed in time, for the Big Bang singularity and the $\mathrm{AH}^{-}$.
The evolution of our model can be visualised more graphically in a 3-d diagram (Fig. 21), which shows the value of the areal radius $R$ at each time $t$ and each value of $M$. Fig. 3 shows the "topographic map" of the surface from Fig. 2] It contains contours ${ }^{6}$ of constant $R$ (the

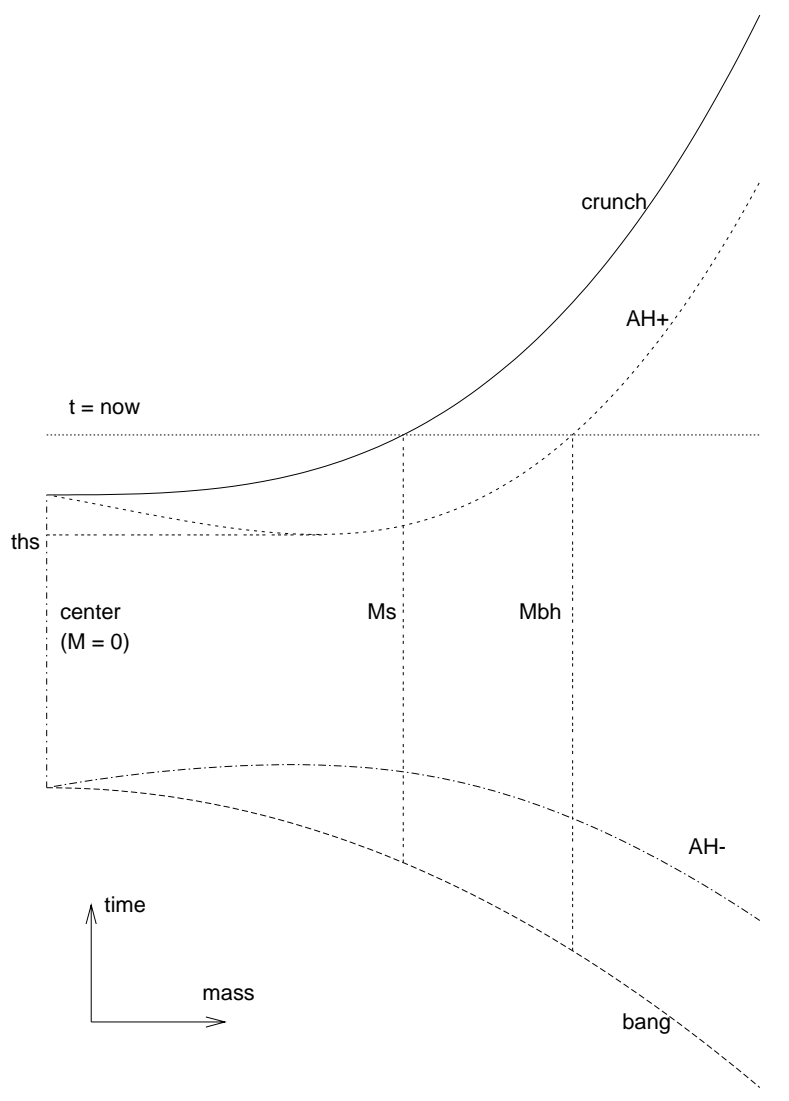

FIG. 1: Evolution leading to a black hole in the $E<0$ L-T model of eqs. 3.30 - 3.31). The final state is defined at the instant $t=t_{2}=$ now. Worldlines of dust particles are vertical straight lines, each has a constant mass-coordinate. Intersections of the line $t=t_{2}$ with the lines representing the Big Crunch and the future apparent horizon determine the masses $M_{S}$ and $M_{B H}$, respectively.

\footnotetext{
${ }^{6}$ Fig. 3 can be used to explain some properties of shell crossings. Since the worldlines of the dust source are constant $M$ lines (i.e. vertical straight lines), they can never intersect in a $t-M$ diagram, even at shell crossings. A shell crossing would show in this picture as a point where a constant- $R$-contour has a horizontal tangent. (No such points exist in this case, because the functions of the L$\mathrm{T}$ model had been chosen appropriately.) The figure graphically explains why, for avoiding shell crossings away from a neck or belly, it is necessary that the Big Bang is a decreasing function of $M$, and the Big Crunch is an increasing function of $M$. The center of symmetry, the Big Crunch and the Big Bang together form the $R=0$ contour. Contours of small constant $R$ must have a similar shape. Hence, if either of the two conditions were
} 
thinner curves) inscribed into Fig. 1 (The other curves are outgoing radial null geodesics, and we shall discuss them further below.)

Rembering that in the $t-M$ diagram, the slopes of the incoming/outgoing radial light rays are each other's mirror images about vertical lines, it is evident from Fig. 3 that $\mathrm{AH}^{ \pm}$are spacelike everywhere except possibly in a neighbourhood of the central line $M=0$ and at future null infinity. From (3.32) and (3.30) we have $e=8 / 3$ and $c=2$ in (3.24), so we find $\bar{B}_{\mathrm{AH}^{-}} \rightarrow 1$ at the origin. Similarly by (3.31) we have $c=3$, and $\bar{B}_{\mathrm{AH}^{+}} \rightarrow 1$. Therefore $\mathrm{AH}^{ \pm}$are both spacelike at the origin too.

Fig. [3also shows several outgoing radial null geodesics.

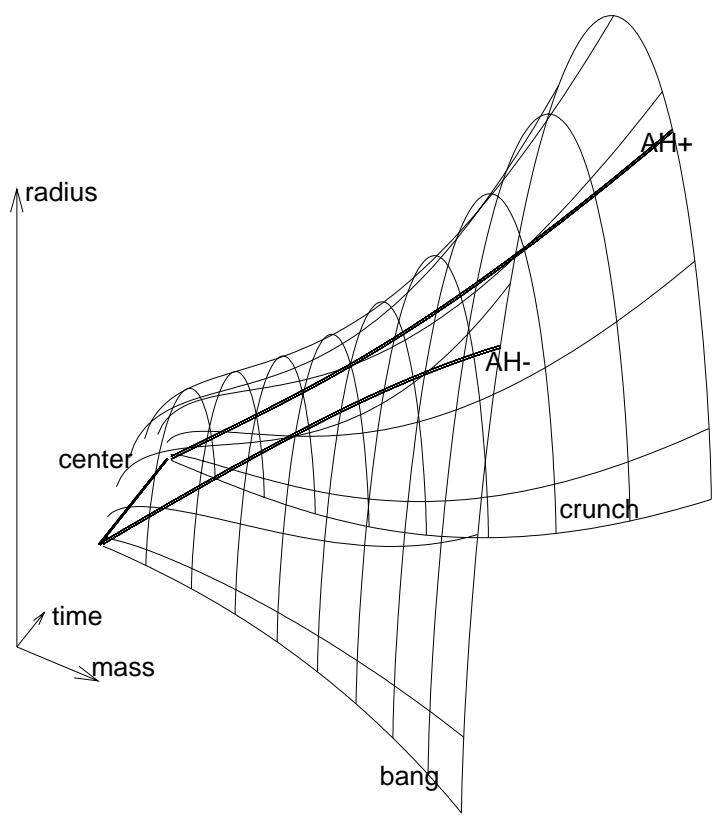

FIG. 2: 3-d graph of the black hole formation process from Fig. 11 the areal radius as a function of $M$ and $t$. Each shell of constant mass evolves in a plane given by $M=$ const. It starts at $R=0$, then gets out of the past apparent horizon $\mathrm{AH}^{-}$, then reaches maximum $R$, then falls into the future apparent horizon $\mathrm{AH}^{+}$, and finally hits the Big Crunch. Note that the surface intersects the $R=0$ plane perpendicularly all along the $R=0$ contour. The apparent horizons are intersections of the $R(M, t)$ surface with the plane $R=2 M$. (Hence, it is easy to figure out how they would look in the $k>0$ Friedmann model, where $t_{B}=$ const, $t_{C}=$ const and all the $\left.R(t)\right|_{M=\text { const. }}$ curves are indentical.

not fulfilled, either the upper branch or the lower branch of some contours would be a non-monotonic function, whose derivative by $M$ would change sign somewhere. At the changeover points, the tangents to the contours would be horizontal, and these would be the shell crossings.
Each geodesic has a vertical tangent at the center. This is a consequence of using $M$ as the radial coordinate. Since $\mathrm{d} t / \mathrm{d} M=\partial R / \partial M / \sqrt{1+2 E}$ on each geodesic and $R \propto M^{2 / 3}$ close to the center, so $\mathrm{d} t / \mathrm{d} M \propto M^{-1 / 3}$ and $\mathrm{d} t / \mathrm{d} M \rightarrow \infty$ as $M \rightarrow 0$. Each geodesic proceeds to higher values of $R$ before it meets the apparent horizon $\mathrm{AH}^{+}$. At $\mathrm{AH}^{+}$, it is tangent to an $R=$ const contour, then proceeds toward smaller $R$ values. The future event horizon consists of those radial null geodesics that approach the $\mathrm{AH}^{+}$asymptotically. In the figure, it lies between the geodesics no 5 and 6 , counted from the lower right corner of the figure; we shall discuss its location in more detail in sec. IIIF3

Geodesic no 5 from the lower right emanates from the center $M=0$, where the Big Bang function has a local maximum. The tangent to the geodesic is horizontal there. This means that the observer receiving it sees the light infinitely redshifted, as in the Friedmann models. Geodesics to the right of this one all begin with a vertical tangent, which implies an infinite blueshift. These observations about redshift and blueshift were first made by Szekeres [39]. Likewise, the geodesics meet the Big Crunch with their tangents being vertical.

By the time the crunch forms at $t=t_{C}(0)$, the future apparent horizon already exists (see Figs. 1 and 3). The shells of progressively greater values of $M$ first go through the $\mathrm{AH}$, and then hit the singularity at $t=t_{C}(M)$. We assume that at the time $t=t_{2}$, the singularity has already accumulated the mass $M_{S}$, while the mass hidden inside the apparent horizon at the same time is $M_{B H}>M_{S}$. Both of them grow with time, but at fixed $t_{2}$ they are constants. From the definitions of $M_{S}$ and $M_{B H}$ it follows that

$$
t_{2}=t_{C}\left(M_{S}\right)=t_{A H+}\left(M_{B H}\right)
$$

\section{Location of the event horizon.}

Now we shall discuss the location of the event horizon in the spacetime model considered in sec. [IIF It will follow that, even though the model has a rather simple geometry, this is quite a complicated task that requires complete knowledge of the whole spacetime, including the null infinity. Hence, in a real Universe, where our knowledge is limited to a relatively small neighbourhood of our past light cone and our past worldline, and the knowledge is mostly incomplete and imprecise, the event horizon simply cannot be located by astronomical observations.

The future event horizon is formed by those null geodesics that fall into the future apparent horizon "as late as possible", i.e. approach it asymptotically. Hence, in order to locate the event horizon, we must issue null 


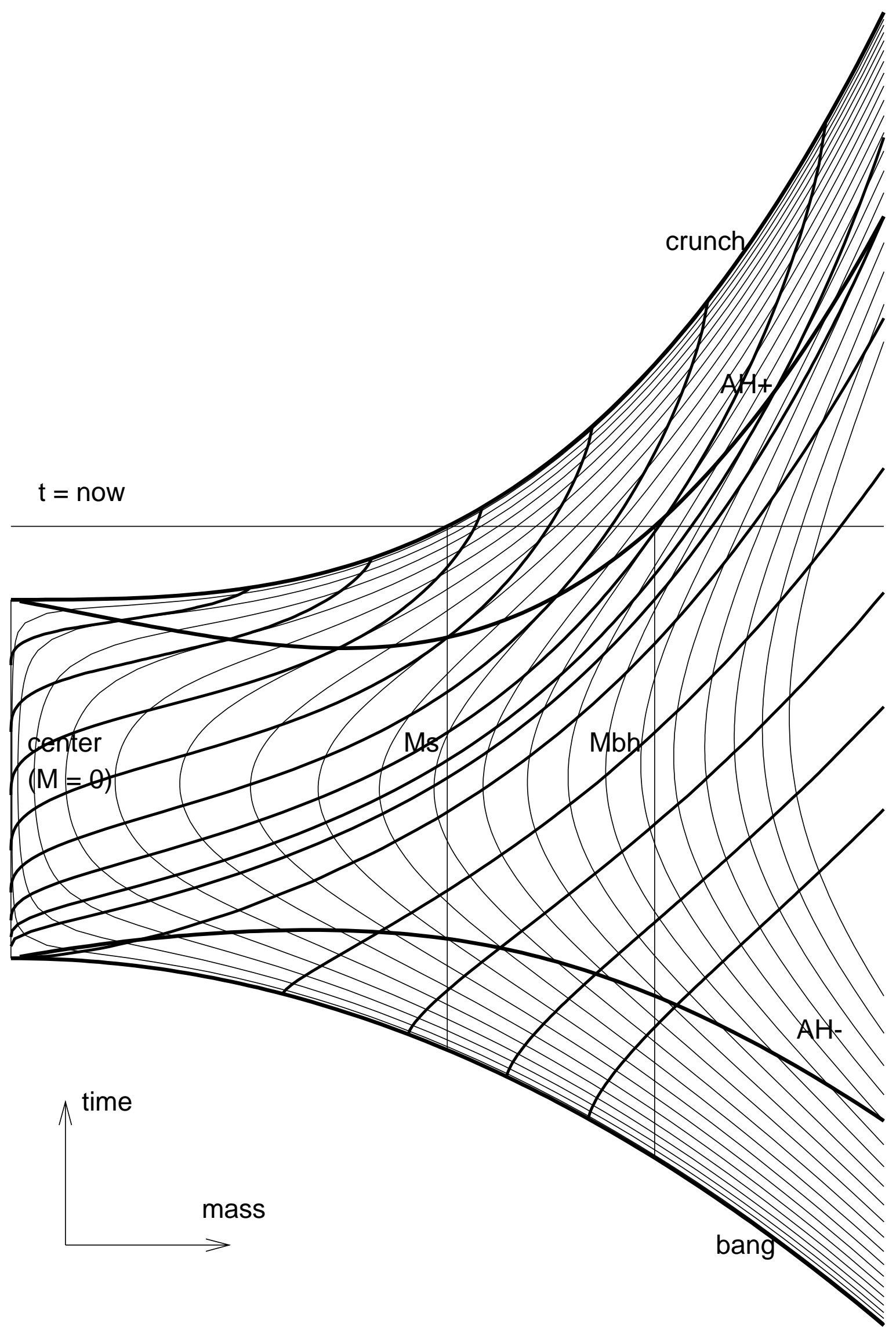

FIG. 3: Contours of constant $R$-value (thinner lines) and outgoing radial null geodesics inscribed into the spacetime diagram of Fig. 1 The $R$-values on consecutive contours differ by always the same amount. 
geodesics backward in time from the "future end point" of the $\mathrm{AH}+$. This cannot be done in the $(M, t)$ coordinates used so far because the spacetime and the $\mathrm{AH}+$ are infinite. Hence, we must first compactify the spacetime. The most convenient compactification for considering null geodesics at a null infinity is, theoretically, a Penrose transform because it spreads the null infinities into finite sets. However, in order to find a Penrose transformation, one must first choose null coordinates, and in the L-T model this has so far proven to be an impossible task, see Ref. [40, 41]. Hence, we will use a less convenient compactification that will squeeze the null infinities into single points in the 2 -dimensional (time - radius) spacetime diagram. It is provided by the transformation

$$
M=\tan (\mu), \quad t=\tan (\tau) .
$$

In these coordinates, the $\mathbb{R}^{1} \times \mathbb{R}_{+}^{1}$ space of Figs. [1 - 3 becomes the finite $[-\pi / 2, \pi / 2] \times[0, \pi / 2]$ rectangle, see Fig. 4 The upper curve in the figure is the Big Crunch singularity; the future apparent horizon runs so close to it that it seems to coincide with it ${ }^{7}$. The horizontal line is the $\tau=$ now line. The lower curve is the Big Bang and the past apparent horizon, again running one on top of the other. The point on the $\tau$-axis where the three lines meet is the image of the $M=0$ line of Fig. 11 squeezed here into a point because of the scale of this figure.

The theoretical method to locate the future event horizon in Fig. 4 would now be to run a radial null geodesic backward in time from the point $(\mu, \tau)=(\pi / 2, \pi / 2)$, i.e. from the image of the future end of the $\mathrm{AH}+$. However, for the most part, the $\mathrm{AH}+$ runs so close to the crunch singularity, and the geodesics intersecting the $\mathrm{AH}+$ are so nearly tangent to $\mathrm{AH}+$, that numerical instabilities crash any such geodesic into the singularity instantly, even if the initial point is chosen well away from $(\pi / 2, \pi / 2)$. This happens all the way down to $\mu=0.5$ at single precision and all the way down to $\mu=1.1$ at double precision. We did succeed, with double precision, only at $\mu=1.0$, and a null geodesic could be traced from there to the center at $\mu=0$. At the scale of Fig. 4 this whole geodesic seems to coincide with the crunch and the $\mathrm{AH}+$. However, it is well visible if one closes in on the image of the area shown in Fig. 11 the closeup is shown in the inset.

Actually, to make sure that we located the event horizon with an acceptable precision, we ran three differ-

\footnotetext{
7 As can be verified from eq. 3.26, the time-difference between the crunch and the $\mathrm{AH}+$ goes to infinity when $M \rightarrow \infty$. However, the ratio of this time-difference to the crunchtime goes to zero, which explains why the two curves in Fig. 4 meet at the image of the infinity. The same is true for the Big Bang and the $\mathrm{AH}^{-}$.
}

ent null geodesics backward in time; one from the point $\mu=1.0, \tau=\tau_{0}:=\tau_{A H+}(1.0)$ right on the apparent horizon, another one from $\mu=1.0, \tau=\tau_{1}$, where $\tau_{1}$ was in the middle between the $\mathrm{AH}+$ and the crunch singularity, and the third one from $\mu=1.0, \tau=\tau_{2}$, where $\tau_{2}$ was below the $\mathrm{AH}+$, with the time-difference $\tau_{0}-\tau_{2}=\tau_{1}-\tau_{0}$. All three ran so close to each other that they actually coalesced along the way and reached the center as one curve; their coincidence in the inset in Fig. 4 is thus not an artefact of scale, but actual coincidence at double precision.

Finally, the event horizon had to be transformed back

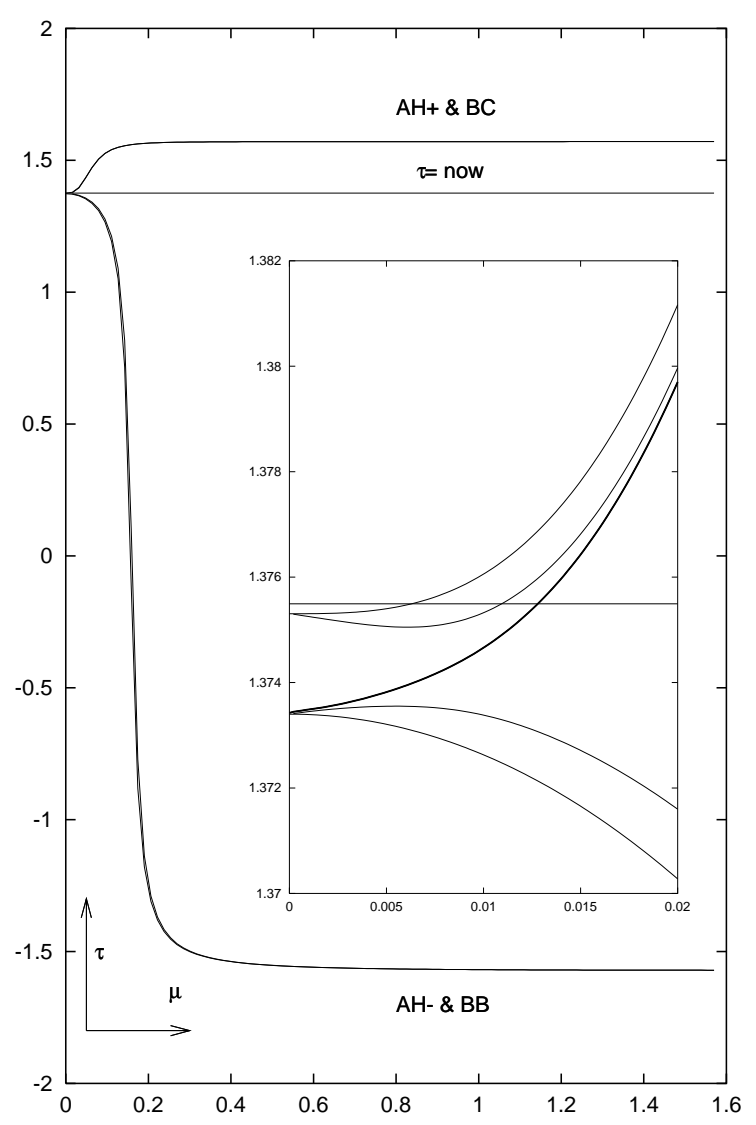

FIG. 4: The spacetime diagram of Fig. 1 compactified according to eq. (3.34). The whole region shown in Fig. 11 is squeezed into the point where the three lines meet at the $\tau$ axis. The worldlines of the dust source would still be vertical straight lines here. The upper curve is the future apparent horizon and the Big Crunch singularity, they seem to coincide at the scale of this picture. The lower curve is the past apparent horizon and the Big Bang singularity, again coinciding only spuriously. The horizontal straight line is the $\tau=$ now time. Inset: a closeup view of the image (in the coordinates $(\mu, \tau))$ of the region shown in Fig. [1] The thicker line is the event horizon. It does not really hit the central point of the Big Bang; the apparent coincidence is just an artefact of the scale. More explanation in the text. 
to the $(M, t)$ - coordinates and written into the frame of the figures 1-3. This is done in Fig. [5 As stated earlier, the event horizon is located between the geodesics no 5 and 6 from the right in Fig. [3 By accident (caused by our choice of numerical values in this example), the $\mathrm{EH}$ hits the center very close to the central point of the Big Bang, but does not coincide with it.

This whole construction should make it evident that there is no chance to locate the event horizon by astronomical observations, even approximately. It only makes sense, in the observational context, to speak about an upper limit on the mass inside the apparent horizon. This is why we identified the observed mass of a black hole with the $\mathrm{AH}$ in sec. IVD

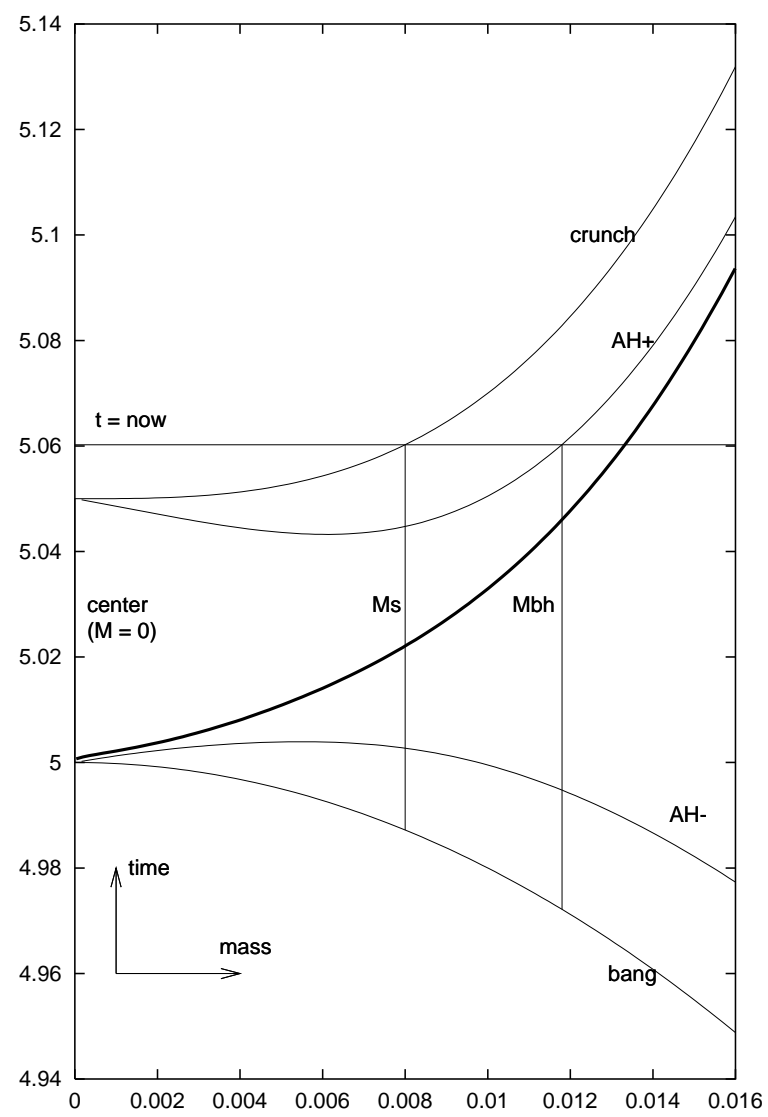

FIG. 5: The event horizon (thicker line) written into the frame of Fig. 11 Its intersection with the $M=0$ axis does not coincide with the central point of the Big Bang, this is only an illusion created by the scale.

\footnotetext{
8 Note that this model in which the event horizon has been found, will actually only be used for the interior of our galaxy model - i.e. for that part of the galaxy that is invisible to outside observers because of its proximity to the black hole horizon. The model of the visible part of the galaxy will be different, and to
}

\section{THE GALAXY PLUS BLACK HOLE FORMATION MODEL}

Our aim is to model the formation of a galaxy with a central black hole, starting from an initial fluctuation at recombination. Our model consists of two parts joined together across a comoving boundary $M=M_{B H}$, with $M_{B H}$ the estimated present day mass inside the black hole horizon. In the exterior part, we take existing observational data for the present day density profile, and the initial fluctuation is made compatible with CMB observations. For the interior, no observational constraints exist, so we propose a couple of possible descriptions, as detailed below. These are both LT models, and represent a collapsing body, and a dense Kruskal-Szekeres wormhole in the sense of [27].

\section{A. The black hole interior}

Astronomical observations do not say anything about that portion of galactic matter that had already fallen inside the apparent horizon by the time the electromagnetic signal that would reach the observer was emitted. What can be seen in the sky are only electromagnetic waves emitted by objects that were still outside the $\mathrm{AH}$ at the time of emission. Consequently, we are not constrained in any way in choosing a model for the matter in the interior of a black hole, except for the need to match it smoothly to a galaxy model.

The term 'black hole' is used in two disctinct ways. Firstly, there is the Schwarzschild-Kruskal-Szekeres black hole, which has the topology of two universes joined by a temporary wormhole, and begins its life as a white hole. This was generalised to a matter-filled version in [27]. Secondly, there is the black hole formed by the collapse of a massive body, which has an ordinary topology without a wormhole. Only in their late stages (after the closure of the wormhole) do these two become essentially the same. Both of these can be reproduced by an L-T model, and we consider them in turn below.

At this point, we must make a digression about the relation between model black holes such as those considered here, and real collapsed objects that are called "black holes" by astronomers. There are two important points to be remembered:

locate the event horizon there, the whole construction (integrating a null geodesic backward in time from future null infinity) would have to be repeated. But, of course, the model of a single galaxy does not extend to infinity. Hence, it hardly makes sense to even speak of an event horizon in this context. 
1. The matter proceeding toward a black hole disappears from the field of view of any real observer before it hits the horizon, whatever horizon is meant (see below). For example, the event horizon is the boundary of the field of view for an observer at future null infinity, i.e. one who is infinitely distant from the black hole and infinitely far into the future. Therefore, the observationally determined "mass of a black hole" is in fact only an upper limit of the mass that has actually fallen within the horizon; the latter can never be measured in reality. Models of black holes allow us to calculate better estimates of that mass, and even if the arithmetic difference between a model calculation and an observational limit is small, it is important to understand the conceptual difference.

2. It is incorrect to speak about the event horizon in the context of observations. We may know where the event horizon is only in a model. In practice, we would have to be able to take into account the future fate of every piece of matter, including those pieces that have been outside our field of view up to now - an obviously impossible task. Worse still, if the real Universe is to recollapse in the future, we might already be inside the event horizon and will never see any signature of it. Hence, the horizons whose signatures we have any chance to see (like disappearance of matter from sight, or a large mass being contained in a small volume) are apparent horizons - they are local entities, detectable in principle at any instant (although with the difficulty mentioned in point 1). Even in our simple model considered in the previous section, it was rather difficult to determine the position of the event horizon, and it could be done only numerically.

In the following we will identify the estimated black hole mass, obtained by fitting a model to present day observations, with $M_{B H}$, the mass within the apparent horizon at time $t_{2}$ (see eq. (3.33)).

\section{B. A collapsed body}

For this example of the interior, we use the model of section $\amalg$ IIF but with different values of the parameters.

9 The Darmois junction conditions for matching an L-T model to itself across a comoving (constant $r$ ) surface only require the
We will assume that $t_{C}(M)$ is an increasing function, to be able to create a black hole, and to prevent shell crossings we assume $t_{B}^{\prime}(M)<0$ in addition. The functions have already been chosen so that the origin conditions are satisfied.

To assure a smooth match to the exterior model, we require the continuity of the $\mathrm{L}-\mathrm{T}$ arbitrary functions and their derivatives ${ }^{9}$ at $M=M_{B H}$. Given equations (3.30) and (3.32), we solve for the constants $a, b, T \& t_{B 0}$, so that $E, t_{B}, \mathrm{~d} E / \mathrm{d} M \& \mathrm{~d} t_{B} / \mathrm{d} M$ are matched at the boundary:

$$
\begin{aligned}
t_{B 0}= & {\left[t_{B}-\frac{M}{2} \frac{\mathrm{d} t_{B}}{\mathrm{~d} M}\right]_{M=M_{B H}}, } \\
b= & -\left[\frac{1}{2 M} \frac{\mathrm{d} t_{B}}{\mathrm{~d} M}\right]_{M=M_{B H}}, \\
T_{0}= & {\left[\frac{M}{6} \frac{\mathrm{d} t_{B}}{\mathrm{~d} M}+\frac{4 \pi M}{3(-2 E)^{3 / 2}}-\frac{2 \pi M^{2}}{(-2 E)^{5 / 2}} \frac{\mathrm{d} E}{\mathrm{~d} M}\right]_{\substack{M=M_{B H} \\
(4.1 \mathrm{c})}}, } \\
a= & {\left[\frac{1}{3 M^{2}} \frac{\mathrm{d} t_{B}}{\mathrm{~d} M}+\frac{2 \pi}{3 M^{2}(-2 E)^{3 / 2}}\right.} \\
& \left.+\frac{2 \pi}{M(-2 E)^{5 / 2}} \frac{\mathrm{d} E}{\mathrm{~d} M}\right]_{M=M_{B H}}
\end{aligned}
$$

\section{A wormhole}

Since we have no way of knowing anything about the matter and spacetime interior to $M_{B H}$, we can equally well fit in a dust-filled wormhole of the Kruskal-Szekeres type, constructed with the L-T metric. The essential requirement is that, at the middle of the wormhole, $M$ must have a minimum value $M_{\min }$, and $E\left(M_{\min }\right)=-1 / 2$. The minimum lifetime (time from past to future singularity) of the wormhole is then $2 \pi M_{\min }$. We choose the following functions:

$$
\begin{aligned}
t_{B} & =t_{B 0}-b\left(M-M_{\min }\right)^{2}, \\
E & =\frac{-M_{\min }}{2 M}+a\left(M-M_{\min }\right) .
\end{aligned}
$$

From these, the conditions for matching to an exterior at some given $M$ value are

matching of $E$ and $t_{B}$ at the same $M$. 


$$
\begin{aligned}
M_{\min } & =\left[M^{2} \frac{\mathrm{d} E}{\mathrm{~d} M}+M\left(1 \pm \sqrt{1+2 E+M^{2}\left(\frac{\mathrm{d} E}{\mathrm{~d} M}\right)^{2}}\right)\right]_{M=M_{B H}}, \\
a & =\left[\frac{1}{2} \frac{\mathrm{d} E}{\mathrm{~d} M}-\frac{1}{2 M}\left(1 \pm \sqrt{1+2 E+M^{2}\left(\frac{\mathrm{d} E}{\mathrm{~d} M}\right)^{2}}\right)\right]_{M=M_{B H}}, \\
b & =\left[\frac{\mathrm{d} t_{B}}{\mathrm{~d} M} /\left\{2 M\left(M \frac{\mathrm{d} E}{\mathrm{~d} M} \pm \sqrt{1+2 E+M^{2}\left(\frac{\mathrm{d} E}{\mathrm{~d} M}\right)^{2}}\right)\right\}\right]_{M=M_{B H}}, \\
t_{B 0} & =\left[t_{B}+\frac{M}{2} \frac{\mathrm{d} t_{B}}{\mathrm{~d} M}\left(M \frac{\mathrm{d} E}{\mathrm{~d} M} \pm \sqrt{1+2 E+M^{2}\left(\frac{\mathrm{d} E}{\mathrm{~d} M}\right)^{2}}\right)\right]_{M=M_{B H}} .
\end{aligned}
$$
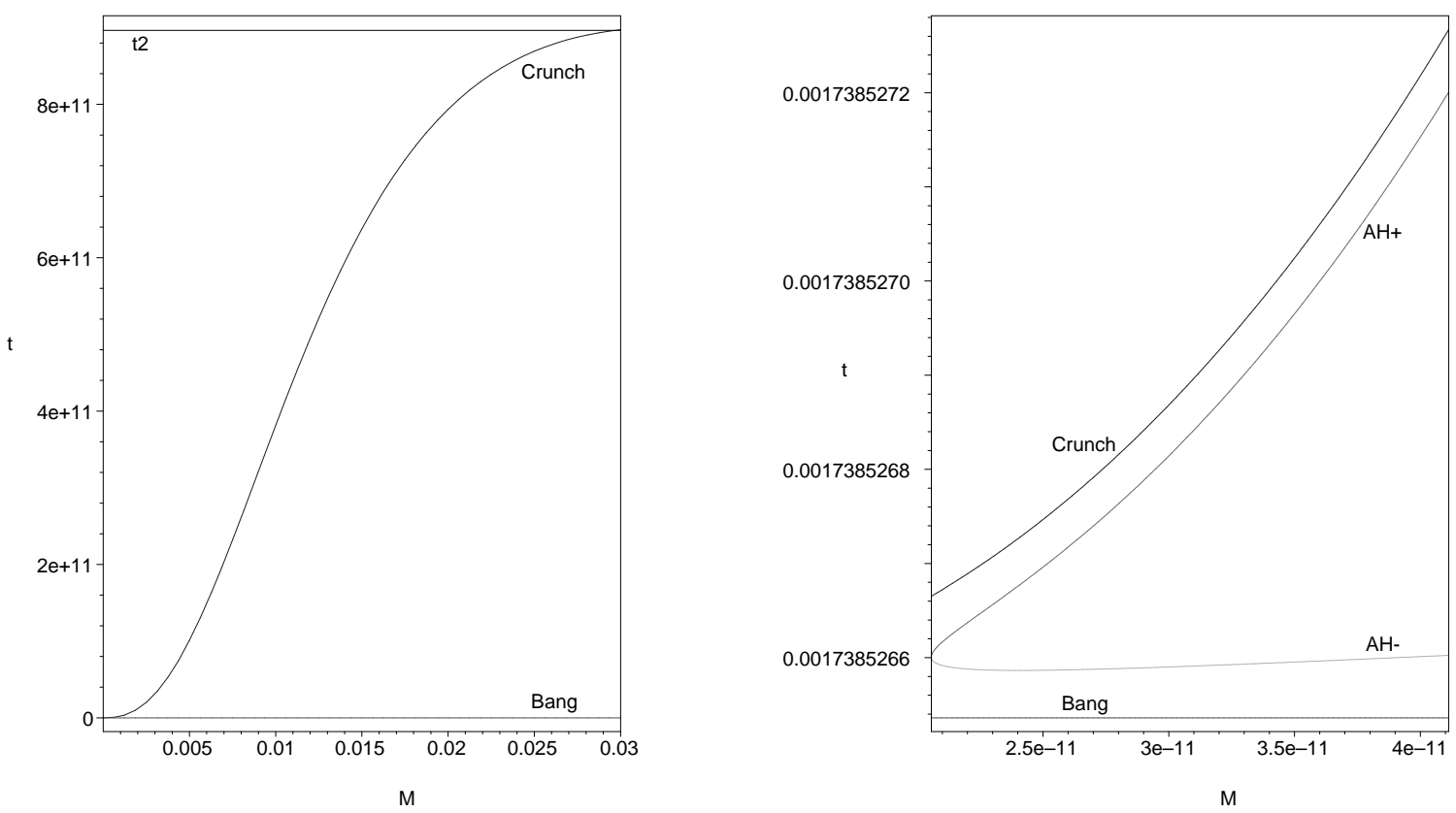

FIG. 6: The L-T model for the wormhole interior of section IVC with parameters (5.2), in geometric units. The second graph shows the neck of the wormhole magnified about $10^{9}$ times. Only on this scale are the apparent horizons distinguishable from the bang \& crunch. The bang does actually curve downwards, but its variation is much less than that of the crunch. The neck is at the left where the mass reaches a minimum $\left(M_{\min }=M_{0}\right)$. The apparent horizons cross in the neck at the moment of maximum expansion. The second sheet is not shown. It could be a mirror image of this sheet, or it could be quite different. The boundary with the exterior model (at $M_{B H}=0.03$ ) is defined to be where $t_{2}=t_{\mathrm{AH}}$. Recombination $t_{1}$ is indistinguishable from the bang in the first graph, and far to the future in the second.

This model was chosen for simplicity, and so is not very flexible. The matching fixes the value of $M_{\min }$, which determines the lifetime of the wormhole. A model with more parameters would allow the wormhole lifetime to be a free parameter. The apparent horizons and singularities for this model with the parameters (5.2) used below are illustrated in Fig. [6]

\section{The exterior galaxy model}

\section{The final density profile}

As our example for the density profile of the final state, we choose the galaxy M87. It is believed to contain a large black hole [42], and the density profile for its outer 
part had been proposed some time ago [43]:

$$
\rho(s)=\rho_{0} /\left(1+b s^{2}+c s^{4}+d s^{6}\right)^{n},
$$

where $\rho_{0}=1.0 \cdot 10^{-25} \mathrm{~g} / \mathrm{cm}^{3}, b=0.9724, c=3.810 \cdot 10^{-3}$, $d=2.753 \cdot 10^{-8}, n=0.59$. The distance from the center, $s$, is measured in arcmin, i.e. it is dimensionless, and is related to the actual distance $r$ by

$$
s=\frac{r}{D} \frac{21600}{\pi}:=r \delta,
$$

where $D$ is the distance from the Sun to the galaxy. (Of course, no galaxy and no real black hole is spherically symmetric, so we cannot model any actual galaxy with the L-T solution. However, we wish to make our illustrative example as close to reality as possible, and this is why we stick to an actual object.) For our purposes, we need density expressed as a function of mass, and a profile that goes to infinity at $r \rightarrow 0$, to allow for the singularity inside the black hole ${ }^{10}$. The mass profile corresponding to (4.4) is not an elementary function. However, it turns out that the following very simple profile is a close approximation to (4.5) in the region considered in Ref. 43. (see Fig. 7):

$$
\rho(r)=\rho_{0} /(\delta r)^{4 / 3},
$$

with the same value of $\rho_{0}$. The corresponding mass distribution is

$$
\widetilde{M}(r)=\frac{12}{5} \pi \rho_{0} r^{5 / 3} \delta^{-4 / 3},
$$

and we make it singular at $r=0$ by adding a constant $M_{S}$ to $\widetilde{M}(r)$ (this is the same $M_{S}$ as defined before the mass that had already fallen into the singularity at $t_{2}$; so far this is still an arbitrary constant):

$$
M(r)=M_{S}+\frac{12}{5} \pi \rho_{0} r^{5 / 3} \delta^{-4 / 3} .
$$

Hence, $r=\left[5\left(M-M_{S}\right) \delta^{4 / 3} / 12 \pi \rho_{0}\right]^{3 / 5}$, and

$$
\rho(M)=(12 \pi / 5)^{4 / 5} \rho_{0}{ }^{9 / 5} \delta^{-12 / 5}\left(M-M_{S}\right)^{-4 / 5} .
$$

In principle, the value of $\rho$ near to $M=M_{B H}$ should be measureable, but in this regime the difference between the Newtonian and L-T definitions of density becomes too pronounced (because of the non-flat geometry). To infer $\rho\left(M_{B H}\right)$ in a sensible way, the results of observations should be consistently reinterpreted within the L-T

10 Even though we will use the interior model near the centre, this requirement assists in joining interior and exterior smoothly. scheme, and such results are not, and will not be available for a long time. Consequently, we will have to give up on this bit of information.

From (2.17), we find the corresponding $R(M)$ via $R^{3}=$ $(3 / 4 \pi) \int_{M_{S}}^{M} \mathrm{~d} x / \rho(x)$, which is

$$
R(M)=\left(\frac{5}{12 \pi \rho_{0}}\right)^{3 / 5} \delta^{4 / 5}\left(M-M_{S}\right)^{3 / 5} .
$$

Now we can determine the constant $M_{S}$ by the requirement that $R=2 M$ at $M=M_{B H}$, i.e.

$$
\left(\frac{5}{12 \pi \rho_{0}}\right)^{3 / 5} \delta^{4 / 5}\left(M_{B H}-M_{S}\right)^{3 / 5}=2 M_{B H}
$$

From here we find

$$
M_{S}=M_{B H}-\frac{12 \pi \rho_{0}}{5 \delta^{4 / 3}}\left(2 M_{B H}\right)^{5 / 3} .
$$

It follows, as it should, that $M_{S}<M_{B H}$. However, this result makes sense only if the $M_{S}$ thus defined is positive.

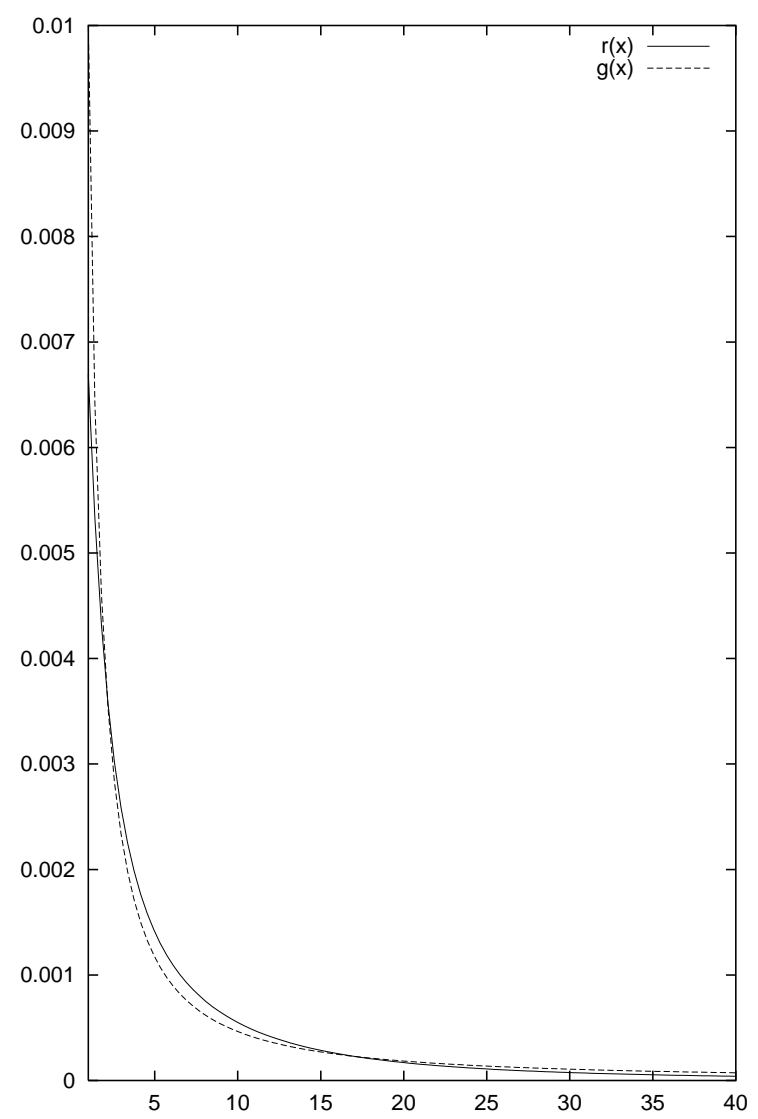

FIG. 7: Comparison of the profile 4.4 (marked as $r(x)$ ) with (4.6) (marked as $g(x)$ ). The $\rho$ on the vertical axis is mass density in units of $10^{-23} \mathrm{~g} / \mathrm{cm}^{3}$, the $x$ on the horizontal axis is the distance from the center in arc minutes, just as in Ref. 43]. The values of parameters are given below eq. (4.4). 
The condition $M_{S}>0$ is equivalent to

$$
\left(\frac{24}{5} \pi \rho_{0}\right)^{3 / 2} \cdot \frac{2 M_{B H}}{\delta^{2}}<1 .
$$

For checking this inequality, all quantities have to be expressed in geometric units. The black hole in M87 is believed to have mass $M_{B H}=3 \cdot 10^{9} M_{\odot}$ [44, 45], and its distance from the Sun is $D=43 \cdot 10^{6}$ light years. In geometric units, with 1 year $=31557600 \mathrm{~s}, c=3 \cdot 10^{9}$ $\mathrm{cm} / \mathrm{s}, G=6.6726 \cdot 10^{-8} \mathrm{~cm}^{3} / \mathrm{g} \cdot \mathrm{s}^{2}$ and $M_{\odot}=1.989 \cdot 10^{33} \mathrm{~g}$, this makes $M_{B H}=4.424 \cdot 10^{14} \mathrm{~cm}, \delta=5.067 \cdot 10^{-22} \mathrm{~cm}^{-1}$, $\rho_{0}=0.741 \cdot 10^{-53} \mathrm{~cm}^{-2}$, and the left-hand side of 4.13) comes out to be $4.075 \cdot 10^{-21}$, which is very safely within the limit.

\section{The initial fluctuation}

In order to define a model uniquely, we only need one more profile for the region $M \geq M_{B H}$, e.g. a density or velocity profile at $t=t_{1}<t_{2}$ or a specific choice of $E(M)$ or $t_{B}(M)$. Apart from the density profile at $t=t_{2}$, there are no other observational constraints in the region $M \geq M_{B H}$ - the time by which galaxies started forming is not well known, and presumably different for each galaxy, nothing is known about the initial density or velocity distribution in the proto-galaxy at that time.

Since the only quantities that are to some degree constrained by the observations are the density and velocity profiles at the recombination epoch, it will be most natural to use these for $t_{1}$. Even so there is a problem: no numerical data are available for amplitudes of the temperature fluctuations of the $\mathrm{CMB}$ radiation at such small scales. We consequently chose a zero velocity fluctuation for one case and a zero density fluctuation for another. However it turned out that the latter was not suitable, since the solution required a collapsing hyperbolic region near $M_{B H}$ in the exterior model.

\section{NUMERICAL EVOLUTION OF THE MODELS}

The programs written for papers I \& II were adapted to facilitate this two-step model construction. First the exterior profiles were used as input to solve numerically for $E(M)$ and $t_{B}(M)$ for $M_{B H} \leq M \leq 1$. The values of $E \&$ $t_{B}$ and their derivatives at $M_{B H}$ were extracted, and the parameters of the interior model calculated from them. Then the functions $E \& t_{B}$ were numerically extended into the interior model, down to $M=0$ or $M=M_{\min }$. From this data, the model evolution was reconstructed using existing programs.
Our first model uses the final density profile of section IVD for the galaxy at time $t_{2}=14 \mathrm{Gyr}$, and a flat initial velocity profile at time $t_{1}=10^{5} \mathrm{y}$, both exterior to $M_{B H}$. The interior of $M_{B H}$ is a black hole formed by collapse, as described by (3.30) and (3.32), with parameters determined by the matching (4.1). Geometric units were chosen such that $10^{11} M_{\odot}$ is the mass unit. In these units, the parameters are:

$$
\begin{aligned}
a=9.0662 & \times 10^{14}, \quad b=0.012409, \\
t_{B 0} & =0.0017385, \quad T_{0}=8.7279 \times 10^{11}
\end{aligned}
$$

The resulting arbitrary functions and the behaviour of the combined model are shown in Figs 8 and 9 Notice that the fluctuations of both density and velocity at recombination are well within $3 \times 10^{-5}$ and $10^{-4}$. The black hole singularity forms at time $T_{0}=13.618 \mathrm{Gyr}$ (since $t_{B 0}=2.7126 \times 10^{-5} \mathrm{y}$ is negligible), so it is 400 million years old by today.

Our second model uses the identical exterior, but the interior is a full Kruskal-Szekeres type black hole containing a temporary 'wormhole', as described by (4.2), with parameters determined by the matching (4.3). The same geometric units were used, and, using the '-' sign in (4.3), the parameters are:

$$
\begin{aligned}
& a=-4.7475 \times 10^{-8}, \quad b=0.012409, \\
& t_{B 0}=0.0017385, \quad M_{\min }=2.0562 \times 10^{-11}
\end{aligned}
$$

(Using the $+\operatorname{sign}$ in (4.2) gives $M_{\min }=0.06>M_{B H}=$ 0.03, which is not acceptable.) Figs [10 and 111 show the arbitrary functions and the behaviour of the combined model for this scenario. Because the exteriors are identical, the fluctuations of density and velocity at recombination $\left(t_{1}\right)$ outside $M_{B H}$ are again well within CMB limits. The wormhole mass (minimum in $M$ ) is $M_{\min }=2.0562 M_{\odot}$, and the future singularity first forms at $T_{0}=6.3613 \times 10^{-5}$ secs after the past singularity. (The future and past black hole singularities are the extension of the crunch and bang into the middle of the wormhole.) The very short lifetime of the wormhole is a consequence of the need for $E$ to go from $-1 / 2$ all the way up to $-1.7669 \times 10^{-9}$ and arrive there with a negative gradient. (Even at constant $M$, (2.8) implies $T\left(M_{B H}\right) / T_{0}>10^{13}$, and the non-zero change in $M$ only increases this factor.) Though models could no doubt be found with quite different wormhole lifetimes, this example very effectively highlights the fact that the nature of the central black hole is essentially unknown. By recombination $\left(t_{1}\right)$, this black hole has accreted ${ }^{11} 246200 M_{\odot}$ within the apparent

\footnotetext{
11 The mass within the $\mathrm{AH}$ at $t_{1}$ is found by numerical root finding, using 3.33 with $t_{1}$ instead of $t_{2}$ and 3.26
} 

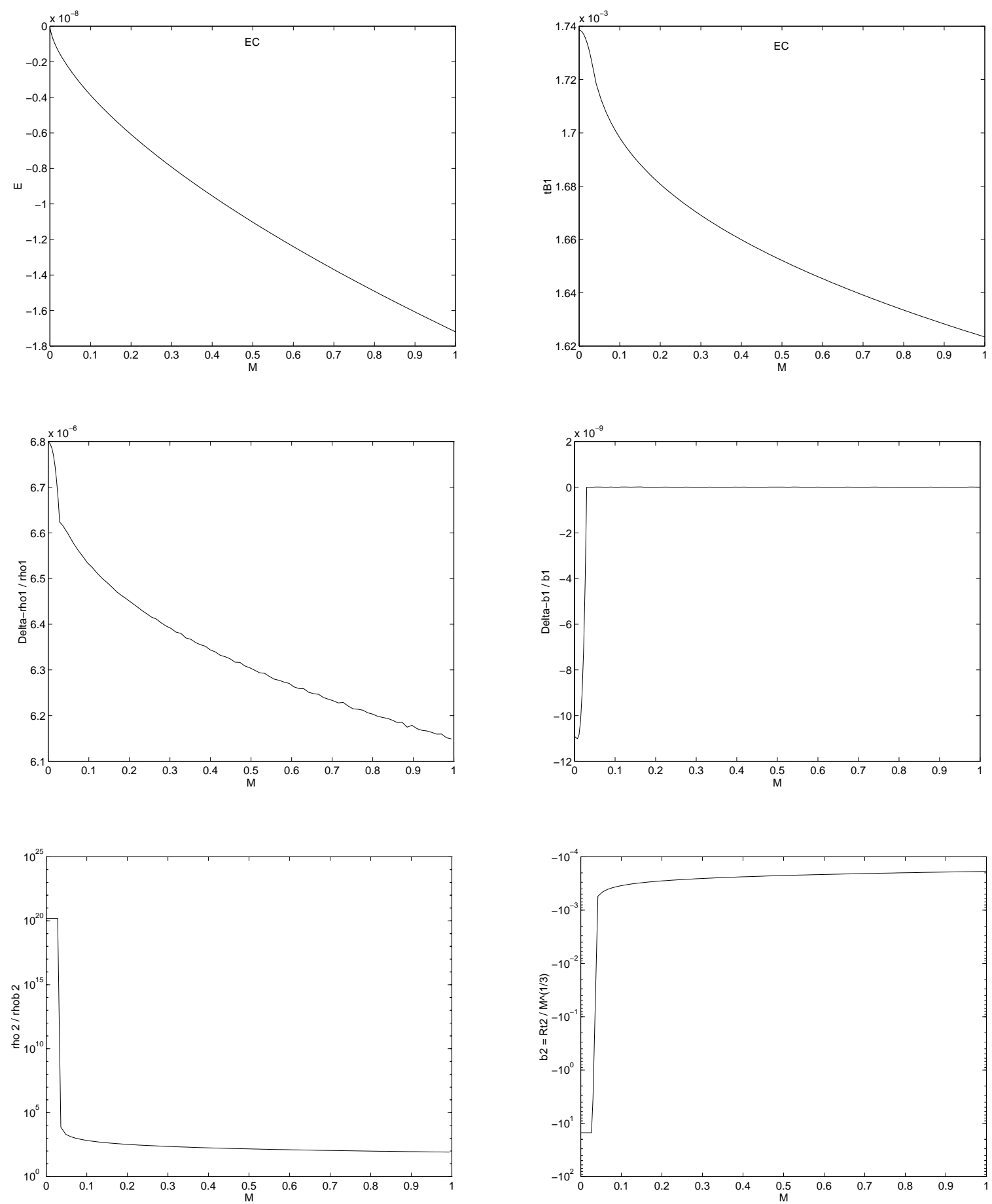

FIG. 8: The L-T model for the formation of a galaxy that develops a central black hole. Shown are the model-defining L-T functions $E(M) \& t_{B}(M)$, the $\rho_{1}(M)$ and $b_{1}(M)$ fluctuations, the $\rho_{2}(M)$ and $b_{2}(M)$ variations. The $b_{2}(M)$ variation is zero, and only very small numerical error shows. Note that the graphs have been clipped at $\log (R)=0, \log (b)=15$ and $\log (\rho)=-5$ (geometric units). The "EC" indicates the range considered is an elliptic region that is collapsing by $t_{2}$.

horizon, which is only $0.0048586 \mathrm{AU}$ across. Any effect this might have on the CMB will not be observable for a long time.

\section{SUMMARY AND CONCLUSIONS}

We have demonstrated the non-linear evolution of an initial density perturbation at recombination into a 

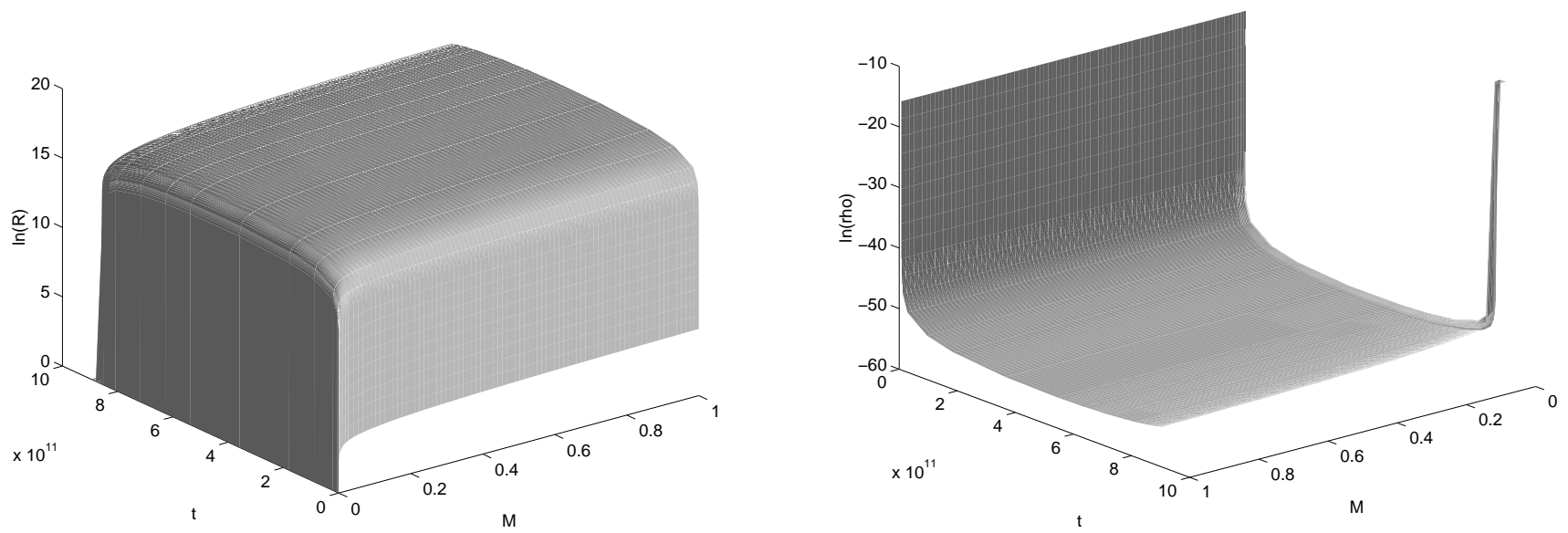

FIG. 9: The evolution of the L-T model for the formation of a galaxy that develops a central black hole. Shown are the evolution of $R(t, M)$ and of $\rho(t, M)$ for run $\mathrm{Vi0} \rho \mathrm{f} 30$. In the $R(t, M)$ graph, the origin $R(t, 0)=0$ is on the left, and expansion to a maximum occurs as time increases towards the "north-west". At the inital time, $t_{1}=$ recombination, $R>0$ except at the origin. In the $\rho(t, M)$ graph the view has been rotated by $180^{\circ}$ relative to the $R(t, M)$ graph for clarity, so that the orgin is on the right and time increases towards the "south-east". By $t_{2}=$ today, the innermost region has collapsed to a black hole of mass $3 \times 10^{9} M_{\odot}$. Note that the graphs have been clipped at $\log (R)=0$ and $\log (\rho)=-5$ (geometric units).

galaxy with a central black hole at the present day, using the spherically symmetric L-T model. This is an application of the methods developed in papers I \& II, in which an initial and a final state - each a density profile or a velocity profile - can be used to derive the arbitrary functions of an L-T model that evolves from one to the other. To correctly describe this process, a relativistic approach is necessary because Newtonian models are inadequate for the description of black holes and their use inevitably leads to conceptual inconsistencies and contradictions. The L-T model is ideal for this purpose, as it has both Schwarzschild and Robertson-Walker limits, and a single model can describe a cosmology containing a black hole.

For the final state at $t_{2}=14 \times 10^{9}$ years $\approx$ today, we chose the model of the mass distribution in the M87 galaxy used in astronomical literature, eqs. (4.4) and (4.5). More exactly, we approximated this mass distribution by a more elementary function whose values do not differ much in the range of interest, eq. (4.6), so that $\rho$ can be calculated as an elementary explicit function of the mass within a sphere of radius $r$. M87 was chosen since it is believed to contain a large black hole around its center, and several of its parameters have been measured or calculated. The inital fluctuation, at the recombination epoch $\left(t_{1}=10^{5}\right.$ years $)$, was chosen to be consistent with limits from the CMB, even though the smallest scales currently observable are much larger than those relevant to galaxy formation. We assumed zero initial velocity perturbation, i.e. a Friedmannian velocity profile. This was sufficient for a unique numerical iden- tification of an L-T model that evolves the given initial state into the given final state. The resulting evolution of the L-T model, was found to be entirely reasonable the implied initial density amplitude was well within the observationally allowed limit of $10^{-5}$, and the model was elliptic and already recollapsing by $t_{2}$ in the whole range of interest. Assuming the presence of a central black hole today, these profiles were taken to be valid for the exterior of the horizon, and a black hole model was smoothly joined on as the interior.

A theoretical model of a black hole must necessarily include the accompanying entities: the final singularity, the apparent horizon and (whenever appropriate) the event horizon. We first discussed the general properties of the apparent horizon. It must necessarily exist in every L-T model. The future apparent horizon $\mathrm{AH}^{+}$ exists in every collapsing model, the past apparent horizon $\mathrm{AH}^{-}$exists in every expanding L-T model; the expanding and recollapsing model has both AHs that can intersect each other only if there exists a neck or belly at which $R^{\prime}=M^{\prime}=E^{\prime}=t_{B}^{\prime}=0, E=-1 / 2$. In every case, the $\mathrm{AH}$ is given by the equation $R=2 M$ that implicitly defines a function $t=t_{A H}(r)$. We discussed whether the AH can be timelike, null or spacelike while going in or out. It turned out that only two cases are excluded: outgoing timelike for $\mathrm{AH}^{+}$and ingoing timelike for $\mathrm{AH}^{-}$. The condition for the $\mathrm{AH}$ to be nontimelike is $\ell \mathrm{d} t_{A H} / \mathrm{d} r \leq \mathrm{d} \mathcal{M} / \mathrm{d} r$, where $\ell= \pm 1$ for $\mathrm{AH}^{\mp}$ and $\mathcal{M}$ is the sum of rest masses of particles within the $r$ sphere. For the Friedmann models, the AH is timelike everywhere. 

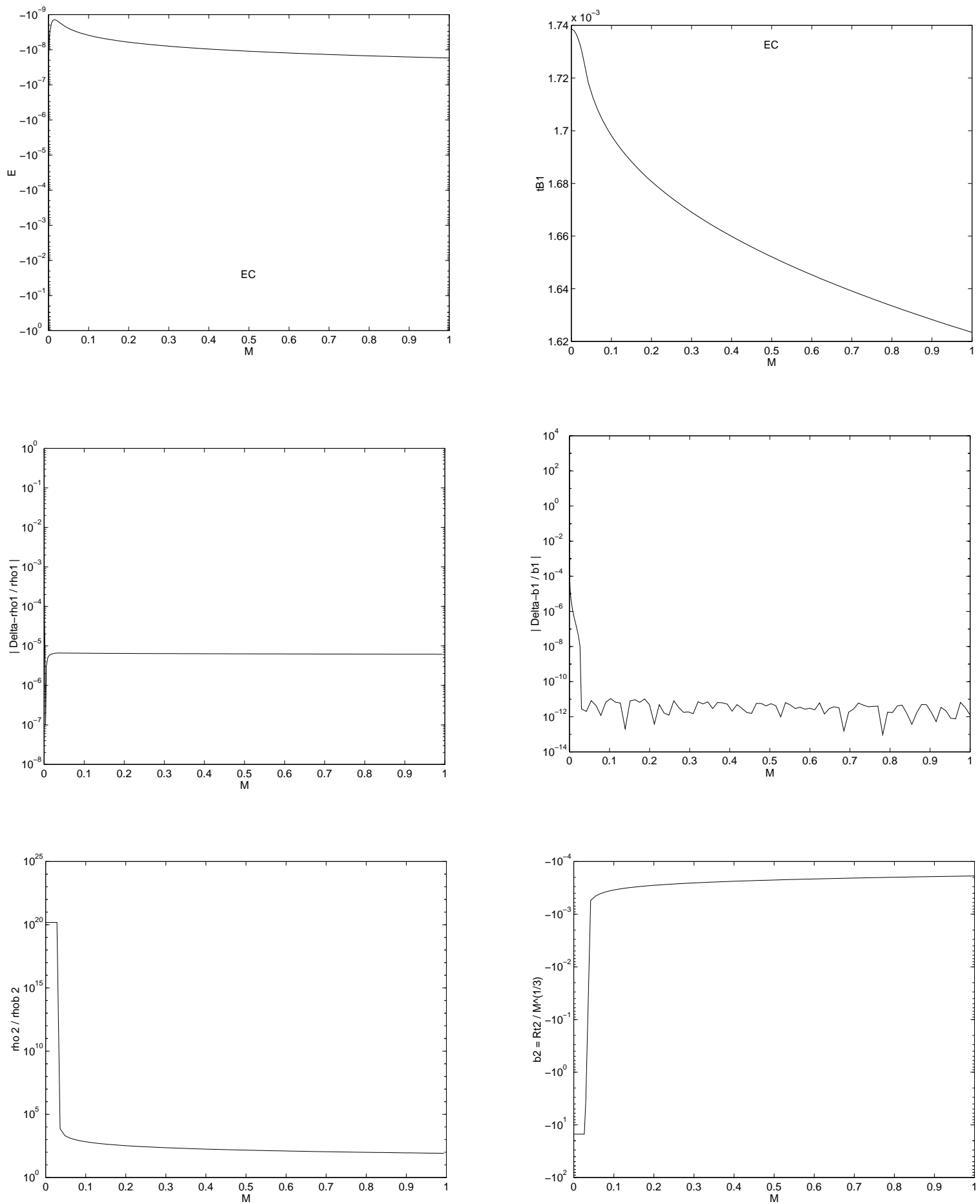

FIG. 10: The L-T model for the formation of a galaxy around a pre-existing central black hole. Shown are the model-defining L-T functions $E(M) \& t_{B}(M)$, the $\rho_{1}(M)$ and $b_{1}(M)$ fluctuations, the $\rho_{2}(M)$ and $b_{2}(M)$ variations. (Again the $b_{2}(M)$ variation is pure numerical error.) Note that the graphs have been clipped at $\log (R)=0, \log (b)=15$ and $\log (\rho)=-5$ (geometric units).

The event horizon (EH) does not always exist; for example it is absent in the $\Lambda=0$ Friedmann models. When it exists, the $\mathrm{EH}^{+}\left(\mathrm{EH}^{-}\right)$is the collection of those futuredirected outgoing (ingoing) radial null geodesics that approach the $\mathrm{AH}^{+}\left(\mathrm{AH}^{-}\right)$asymptotically as $t \rightarrow+\infty$ $(t \rightarrow-\infty)$. The equation of null geodesics is in general intractable by exact methods, and so locating the EH inevitably requires numerical integration. This can only 

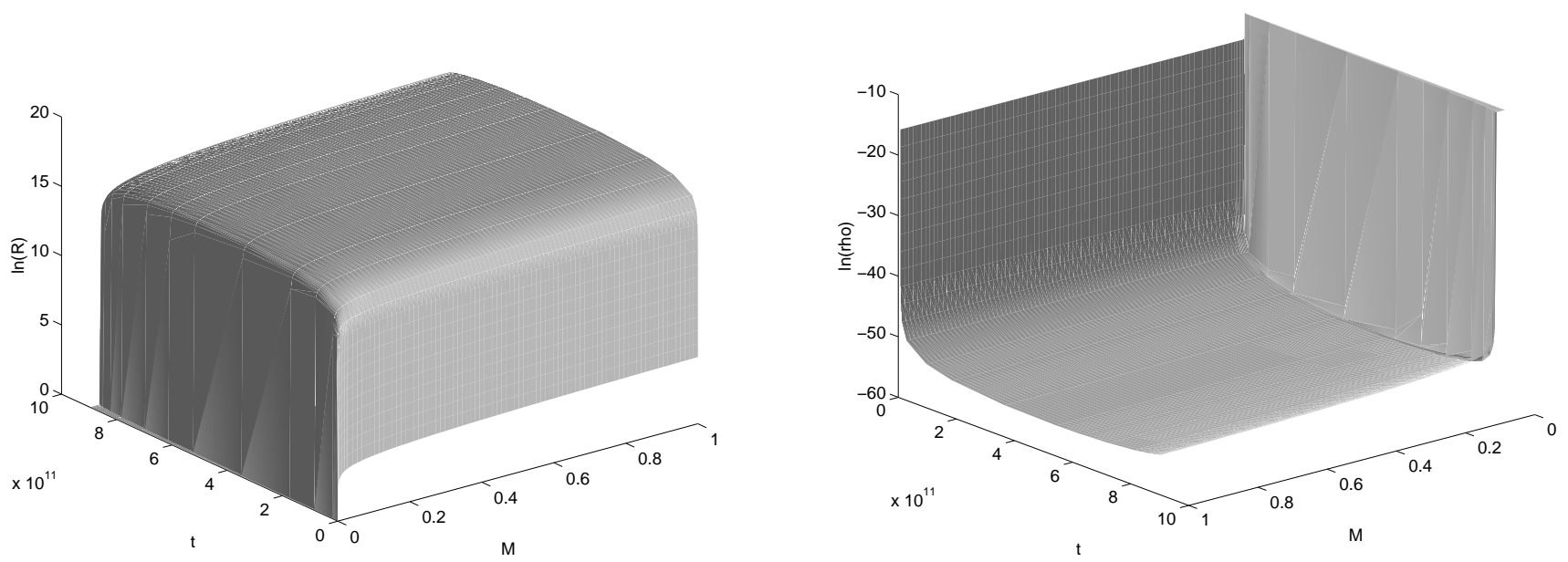

FIG. 11: The evolution of the L-T model for the formation of a galaxy around a pre-existing central black hole. Shown are the evolution of $R(t, M)$ and of $\rho(t, M)$ for run $\mathrm{Vi} 0 \rho \mathrm{f} 30$. In the $R(t, M)$ graph, on the left there is no origin, rather a wormhole with a tiny lifetime re-collapses to zero size. The thin flat wedge along the left is the growing singularity. (The apparent ripples are small numerical variations overemphasised by the graphics program.) In the $\rho(t, M)$ graph, again with the view rotated by $180^{\circ}$ relative to the $R(t, M)$ graph, the density diverges towards the singularity, and the flat wedge represents the part that has already collapsed. By $t_{2}=$ today, the black hole mass has increased to $3 \times 10^{9} M_{\odot}$. Note that the graphs have been clipped at $\log (R)=0$ and $\log (\rho)=-5$ (geometric units).

be done case by case, for specific forms of the L-T functions $E(r)$ and $t_{B}(r)$. With a space of infinite volume, the numerical identification of the EH can most easily be done after the spacetime is compactified so that the future (past) edge of the $\mathrm{AH}^{+}\left(\mathrm{AH}^{-}\right)$has finite time and radial coordinates.

Then we calculated all the characteristic quantities of a black hole (the past and future singularity, both $\mathrm{AH}^{-} \mathrm{s}$, radial null geodesics and the $\mathrm{EH}+$ ) in a simple illustrative toy model with $E<0$ and displayed them in spacetime diagrams. The model is recollapsing, has infinite total mass and volume, and a duration between bang and crunch that is finite at every finite mass, but goes to infinity as $M \rightarrow \infty$ and $E \rightarrow 0$.

Since no observational data exist (and, presumably, will not exist for a long time) concerning the interior of the horizon, two distinct forms of this central black hole - both L-T models - were considered, firstly a condensation that collapses to a singularity, and secondly a full Schwarzschild-Kruskal-Szekeres type wormhole topology. The parameters of these models were determined by matching their L-T functions to those of the exterior galaxy-forming model. In the case of collapse to a black hole, the central singularity is $\approx 4 \times 10^{8}$ years old by now. For the wormhole case, the final singularity forms almost immediately after the Big Bang $\left(6.36 \times 10^{-5} \mathrm{sec}.\right)$, and is by today about as old as the matter in the galaxy. In this model, the black hole accretes mass very fast into a very small volume, so that by recombination it had swallowed up $246380 M_{\odot}$ in a region of diameter $0.00486 \mathrm{AU}$. However, all the numbers are strongly model-dependent, and there are no reliable observational constraints for model selection. The initial black hole is too small to have an observable effect on the CMB. Therefore we find both types of black hole are possible. Perhaps small black holes, that avoid evaporation by rapid accretion, may seed galaxy formation.

In fact, existing observational data does not have the resolution to constrain the initial data for our model. For example, as shown in Paper I, the perturbations of isotropy of the CMB temperature corresponding to single galaxies should have the angular size of $\approx 4 \times 10^{-3}$ degrees, while the most precise current measurements have the resolution of $0.2^{\circ}$.

Then, in the vicinity of the apparent horizon, the geometry of spacetime becomes measureably nonMinkowskian, while all the observational data available on mass distribution within galaxies were calculated by purely Euclidean reduction methods. We also stressed that what can be inferred from observations is only the upper limit of the mass inside the apparent horizon at a given time. It does not make sense to even speak of an event horizon in the observational context.

In view of the paucity of data, our approach was the first exploratory step into an uncharted territory rather than an actual model to be compared with observations.

The main limitation of the spherically symmetric L-T model is the lack of rotation, which slows collapse and stablises structures. Thus the model is good for much of the evolution into the non-linear regime, but becomes 
less realistic as collapse sets in.

Our results show that the L-T model is a very useful tool for this kind of investigation. However, for its parameters to be fine-tuned to results of observations, the observational data would have to be re-interpreted against the background of the $\mathrm{L}-\mathrm{T}$ geometries.

\section{Acknowledgments}

The research of AK was supported by the Polish Research Committee grant no 2 P03B 12 724. CH thanks the South African National Research Foundation for a grant.

\section{APPENDIX A: THE FRIEDMANN LIMIT}

We briefly specialize the above results to the Friedmann limit, where

$$
\begin{aligned}
M=M_{0} r^{3}, \quad 2 E & =-k r^{2}, \\
& t_{B}=\text { const }, \quad R=r S(t),
\end{aligned}
$$

$M_{0}>0$ and $k>0$ being arbitrary constants, and $S(t)$ being the scale factor. The apparent horizon, where $R=$ $2 M$, has the equation

$$
S(t)=2 M_{0} r^{2}
$$

so by (3.13), (3.3) and (3.14) we obtain

$$
\begin{gathered}
B=1-\frac{2 M^{\prime}}{R^{\prime}}=-2, \\
t_{n}^{\prime}=j \frac{S}{\sqrt{1-k r^{2}}}, \\
t_{\mathrm{AH}^{ \pm}}^{\prime}=\ell \frac{4 M_{0} r^{2}}{\sqrt{1-k r^{2}}}=2 \ell j\left(t_{n}^{\prime}\right)_{\mathrm{AH}^{ \pm}} .
\end{gathered}
$$

Hence, in the Friedmann limit both branches of the $\mathrm{AH}$ are entirely timelike (outgoing in the expansion phase, incoming in the collapse phase) and monotonic with $r$. The derivatives $t_{A H \pm}^{\prime}$ and $t_{n}^{\prime}$ seem to become infinite at $r=1 / \sqrt{k}$. This is a coordinate effect. As seen from the metric $\mathrm{d} s^{2}=\mathrm{d} t^{2}-S^{2}(t)\left(\frac{\mathrm{d} r^{2}}{1-k r^{2}}+r^{2} \mathrm{~d} \omega^{2}\right)$, there is a coordinate singularity at $r=1 / \sqrt{k}$. Both derivatives become finite when the coordinates are changed so that e.g. $r=\sin r^{\prime}$. The quantity $B$ in eq. (A3) does not depend on the choice of $r$.

For a completely general Robertson-Walker model (i.e not just $p=0$ ), repeating the whole reasoning, we obtain for the slope:

$$
B=\frac{\left(\dot{S}^{2}+k\right)}{S \ddot{S}},
$$

which, after making use of the $\Lambda=0$ Einstein equations, is equivalent to

$$
B=\frac{-2 \rho}{3 p+\rho} .
$$

This makes the AHs timelike for $1 / 3>p / \rho>-1 / 3$, but spacelike for $p / \rho>1 / 3$, or for $p / \rho<-1$. (Note that $B=0$ requires $\rho=0$ or divergent $p$.) Hence, it cannot be decided whether the $\mathrm{AH}$ is timelike or not without knowing the precise shape of the function $S(t)$ (i.e. knowing the equation of state). It can only be said that as long as the source in the Einstein equations is ordinary matter known from laboratory (no cosmological constant or other self-accelerating medium), we will have $\ddot{S}<0$. Consequently, by (A6), $B<0$, which means that the $\mathrm{AH}$ will be outgoing in the expansion phase and incoming in the collapse phase.
[1] "The Supermassive Black Hole at the Galactic Center", F. Melia, H. Falcke, Ann. Rev. Astron. Astrophys. 39, 309-52 (2001)

[2] M. Haehnelt, P. Natarajan and M.J. Rees Mon. Not. Roy. Astron. Soc. 300, 817 (1998).

[3] A. Franceschini, S. Vercellone and A.C. Fabian Mon. Not. Roy. Astron. Soc. 297, 817 (1998).

[4] A. Cattaneo, M. Haehnelt and M.J. Rees Mon. Not. Roy. Astron. Soc. 308, 77 (1999).

[5] F. Leeuwin and E. Athanassoula Mon. Not. Roy. Astron. Soc. 317, 79 (2000).

[6] M.G. Haehnelt and G. Kauffmann Mon. Not. Roy. Astron. Soc. 318, L35 (2000).
[7] W. Maciejewski and J. Binney Mon. Not. Roy. Astron. Soc. 323, 831 (2001).

[8] R.J. McLure and J.S. Dunlop Mon. Not. Roy. Astron. Soc. 327, 199 (2001).

[9] M. Vestergaard Astrophys. J. 571, 733-52 (2002).

[10] C.A. Onken and B.M. Peterson Astrophys. J. 527, 746-52 (2002).

[11] C.A. Onken, B.M. Peterson, M. Dietrich, A. Robinson and I.M. Salamanca Astrophys. J. 585, 121-7 (2003).

[12] M. Milosavljevi, D. Meritt, A. Rest and F.C. van den Bosch Mon. Not. Roy. Astron. Soc. 331, L51 (2002).

[13] J. Chiang and O. Blaes Astrophys. J. 586, 97-111 (2003).

[14] A.V. Filippenko and L.C. Ho Astrophys. J. 588, L13-16 
(2003).

[15] F.K. Liu, X-B Wu and S.L. Cao Mon. Not. Roy. Astron. Soc. 340, 411 (2003).

[16] "Active Galactic Nuclei and Related Phenomena", IAU symposium S-194, Eds. Y. Terzian, D. Weedman and E. Khachikian (Astronomical Society of the Pacific, 1999), ISBN: 1-58381-008-0

[17] "The Central Parsecs of the Galaxy - Galactic Center Workshop", ASP Conference 186, Eds. H. Falke, A. Cotera, W.J. Duschl, F. Melia and M.J. Rieke (Astronomical Society of the Pacific, 1999), ISBN: 1-58381-0129

[18] "Galaxies and their Constituents at the Highest Angular Resolutions", IAU symposium S-205, Eds. R.T. Schilizzi, S.N. Vogel, F. Paresce and M.S. Elvis (Astronomical Society of the Pacific, 2001), ISBN: 1-58381-66-8

[19] M. C. Begelman, Science 300, 1898 (2003).

[20] Paper I A. Krasiński and C. Hellaby, Phys. Rev. D 65, 023501 (2002).

[21] Paper II A. Krasiński and C. Hellaby, Phys. Rev. D, to be published (2004), gr-qc/0303016

[22] G. Lemaître, Ann. Soc. Sci. Bruxelles A53, 51 (1933); reprinted in Gen. Rel. Grav. 29, 641 (1997).

[23] R. C. Tolman, Proc. Nat. Acad. Sci. USA 20, 169 (1934); reprinted in Gen. Rel. Grav. 29, 935 (1997).

[24] A. Krasiński, "Inhomogeneous Cosmological Models", Cambridge U P (1997), ISBN 0521481805.

[25] N. Mustapha and C. Hellaby, Gen. Rel. Grav. 33, 455-77 (2001).

[26] C Hellaby, K. Lake, Astrophys. J. 290, 381 (1985) [+ erratum: Astrophys. J. 300, 461 (1985)].
[27] C. Hellaby, Class. Q. Grav. 4, 635 (1987).

[28] H. Bondi, Mon. Not. Roy. Astr. Soc. 107, 410 (1947) [reprinted in Gen. Rel. Grav. 31, 1783 (1999)].

[29] D. M. Eardley, L. Smarr, Phys. Rev. D 19, 2239 (1979).

[30] D. Christodoulou Commun. Math. Phys 93, 171 (1984).

[31] R. P. A. C. Newman, Class. Q. Grav. 3, 527 (1986).

[32] R. P. A. C. Newman, Ann. Phys 182, 112 (1988).

[33] V. Gorini, G. Grillo, M. Pelizza, Phys. Lett. A135, 154 (1989).

[34] J. P. S. Lemos, Phys. Lett. A158, 279 (1991).

[35] I. H. Dwivedi, P. S. Joshi Class. Q. Grav. 9, L69 (1992).

[36] P. S. Joshi, I. H. Dwivedi Phys. Rev. D 47, 5357 (1993).

[37] P. S. Joshi Global Aspects in Gravitation and Cosmology. Clarendon Press, Oxford, pp. 242 - 255 (1993).

[38] W. B. Bonnor, Mon. Not. Roy. Astr. Soc. 215, 597 (1985).

[39] P. Szekeres, in: Gravitational Radiation, Collapsed Objects Exact Solutions. Edited by C. Edwards. Springer (Lecture notes in physics, vol. 124), New York (1980), p. 477.

[40] W. R. Stoeger, G. F. R. Ellis and S. L. Nel, Class. Q. Grav. 9, 509 (1992).

[41] C. Hellaby, J. Math. Phys. 37, 2892-905 (1996).

[42] http://hyperphysics.phy-astr.gsu.edu/hbase/astro/m87.html

[43] D. Fabricant, M. Lecar, P. Gorenstein, Astrophys. J. 241, 552 (1980).

[44] T. DiMatteo, S. W. Allen, A. C. Fabian, A. S. Wilson and A. J. Young, Astrophys. J. 582, 133-140 (2003).

[45] F. Macchetto, A. Marconi and D.J. Axon Astrophys. J. 489, 579-600 (1997). 